\title{
Facet-Dependent Stability of Near-Surface Oxygen Vacancies and Excess Charge Localization at $\mathrm{CeO}_{2}$ Surfaces
}

\author{
Patricia Pérez-Bailac ${ }^{\dagger, \perp}$, Pablo G. Lustemberg ${ }^{\dagger, S,}{ }^{*}$, and M. Verónica Ganduglia-Pirovano ${ }^{\dagger}$ \\ ${ }^{\dagger}$ Instituto de Catálisis y Petroleoquímica (ICP-CSIC), C/Marie Curie 2, 28049 Madrid, Spain \\ ${ }^{\perp}$ PhD Programme in Applied Chemistry, Doctoral School, Universidad Autónoma de Madrid, \\ C/Francisco Tomás y Valiente 2, 28049 Ciudad Universitaria de Cantoblanco, Madrid, Spain \\ SInstituto de Física Rosario (IFIR-CONICET) and Universidad Nacional de Rosario (UNR), Ocampo y \\ Esmeralda, S2000EKF Rosario, Santa Fe, Argentina \\ E-mail: p.lustemberg@csic.es
}

\begin{abstract}
To study the dependence of the relative stability of surface $\left(V^{A}\right)$ and subsurface $\left(V^{B}\right)$ oxygen vacancies with the crystal facet of $\mathrm{CeO}_{2}$, the reduced (100), (110) and (111) surfaces, with two different concentrations of vacancies, were investigated by means of density functional theory $(D F T+U)$ calculations. The results show that the trend in the near-surface vacancy formation energies for comparable vacancy spacings, i.e. $(110)<(100)<(111)$, does not follow that in the surface stability of the facets, i.e. $(111)<(110)<(100)$. The results also revealed that the preference of vacancies for surface or subsurface sites, as well as the preferred location of the associated $\mathrm{Ce}^{3+}$ polarons, are facet and concentration dependent. At the higher vacancy concentration, the $V^{A}$ is more stable than the $V^{B}$ at the (110) facet whereas at the (111), it is the other way around, and at the (100) facet, both the $V^{A}$ and the $V^{B}$ have similar stability. The stability of the $V^{A}$ vacancies, compared to that of the $\mathrm{V}^{\mathrm{B}}$, is accentuated as the concentration decreases. Nearest neighbor polarons to the vacant sites are only observed for the less densely packed (110) and (100) facets. These findings are rationalized in terms of the packing density of the facets, the lattice relaxation effects induced by vacancy formation and the localization of the excess charge, and the repulsive $\mathrm{Ce}^{3+}-\mathrm{Ce}^{3+}$ interactions.
\end{abstract}

Keywords: cerium oxide surfaces, facet-dependent stability, oxygen vacancies, lattice relaxations, excess charge localization

\section{INTRODUCTION}

Cerium dioxide $\left(\mathrm{CeO}_{2}\right)$ and $\mathrm{CeO}_{2}$-based materials have been extensively studied for their excellent oxygen storage capacity (OSC), redox catalytic properties [1], and recently for strong 
metal-support interaction effects in ceria-supported metal nanoparticles [2]. The unique role of ceria has been confirmed in many catalytic applications, including advanced car converters for exhaust gas pollution control [3], hydrogen production and fuel cells [4-6], $\mathrm{CH}_{4}$ conversion to fuels and chemicals [7-11], soot oxidation [12], $\mathrm{CO}$ oxidation to $\mathrm{CO}_{2}$ [13], oxygen sensors [14] and complex organic reactions [15], as well as it holds promise for biological applications [16, 17] Ceria nanoparticles with controlled morphologies, such as nanooctahedra ((111) facet), nanocubes ((100) facet), and nanorods ((100) and (110) facets), are fabricated [18], and their chemisorption and reactivity properties investigated [19-25], with the caveat that under reaction conditions, ceria facets can undergo restructuring [26-31]. There is ample evidence of the facet-dependent properties of ceria nanoparticles, many of which are related to differences in the ease with which oxygen vacancies can be created, which is accompanied by localization of the excess charge on cerium ions, driving the $\mathrm{Ce}^{4+} \rightarrow \mathrm{Ce}^{3+}$ reduction and the formation of two $\mathrm{Ce}^{3+}$ polarons per vacancy [32-35]. For ceria, the in-depth understanding of the facet-dependent oxygen vacancy structure provides a means to interpret the system functionality. To date, in this context, the reduced (111) facet has been the most experimentally and theoretically studied, and the stability of near-surface oxygen vacancies and the excess charge localization have been addressed [36-57]. However, similar studies for the other low-index reduced (110) and (100) facets are relatively scarce and limited to theoretical investigations $[42,44,46,47,58-62]$, within which, those that study the stability of vacancies in the subsurface are even more scarce $[63,64]$. Moreover, in the cases of the less studied (110) and (100) facets, there is a lack of consistency on the relative stability of surface and subsurface vacancies and on which are the preferred cation sites for the localization of the $\mathrm{Ce}^{3+}$ cations created upon oxygen vacancy formation.

With regard to theoretical studies on the reduced (111) facet, the computational methodology varies, but there is general consensus that subsurface vacancies are more stable than surface ones and, for some time now, that the $\mathrm{Ce}^{3+}$ ions are preferably located in the outermost cationic plane in sites not adjacent to the vacancies, as much as possible $[43-45,49,51-54,56,63$, 65], in full accord with experimental results $[33,37,38]$. Therefore, the recent result by Wu et al. [57] which reports that $\mathrm{Ce}^{3+}$ are first neighbors to both surface and subsurface vacancies, is striking. Vacancy-induced lattice relaxation effects were used to explain the preferred localization of both vacancies and excess charge $[43,49,51]$.

Nolan et al. [42] were among the first to study surface oxygen vacancies at the three lowindex facets of ceria with the $\mathrm{Ce}^{3+}$ ions in nearest-neighbor positions to the vacancies, by means of 
density functional theory (DFT+U) calculations, providing the $(110)<(100)<(111)$ trend for the vacancy formation energy. Moreover, Nolan et al. [42] noted that the trend did not follow the one of the stability of the pure surfaces, namely, $(100)<(110)<(111)$, however, no explanation was provided.

The theoretical understanding of the structures of near-surface oxygen vacancies at the (110) facet is shallower than at the (111) facet $[32,33,42,44,58,59,61,63,66]$ with hardly any consideration of subsurface vacancies [63]. Initially, Nolan et al. [46] and Fabris et al. [44] performed $\mathrm{DFT}+\mathrm{U}$ calculations for a surface vacancy at the surface with the excess charge localized on two $\mathrm{Ce}^{3+}$ adjacent to the vacancy. Scanlon et al. [61] later reported the higher stability (by about $0.3 \mathrm{eV}$ with $\mathrm{DFT}+\mathrm{U},(2 \times 2)$ unit cell) of a configuration for which both $\mathrm{Ce}^{3+}$ are next to the vacancy, but one is in the surface layer and the other in the layer below. Afterwards, Nolan confirmed the higher stability of that configuration using hybrid-DFT, [58] and Song et al. [65] also reported that it was the most stable with DFT+U. However, Kullgren et al. [59] revealed that another configuration with both $\mathrm{Ce}^{3+}$ in the outermost ceria layer, one adjacent to the vacancy and the other one in a next-nearest neighbor position, is energetically preferred compared to both the one with two $\mathrm{Ce}^{3+}$ adjacent to the vacancy in the surface layer and the one with one of the nearest-neighbors $\mathrm{Ce}^{3+}$ in the layer below (by about 0.7 and $0.3 \mathrm{eV}$, respectively, with $\mathrm{DFT}+\mathrm{U},(2 \times 2)$ unit cell). The high stability of the last-reported vacancy structure is maintained for different unit cell sizes, although the energy for creating the vacancy can vary in a range of $0.5 \mathrm{eV}$ [59]. The recently reported $\mathrm{Ce}^{3+}$ distribution for a surface vacancy at the (110) surface by Wu et al. [63] is consistent with what was found by Kullgren et al. [59] Moreover, a greater stability of surface vacancies than subsurface ones has been recently reported [63] with the subsurface vacancy having both $\mathrm{Ce}^{3+}$ adjacent to the vacancy, but neither of them in the outermost layer but in deeper layers, what is surprising. The dependence of the relative stability of vacancies near the (110) surface with the unit cell size was not considered.

The reduced (100) facet is probably the least studied [34, 42, 58, 63, 64], Nolan et al. [42, 58] reported that the stable surface vacancy has two $\mathrm{Ce}^{3+}$ adjacent to the vacancy (with DFT+U and hybrid-DFT, $p(2 \times 2)$ unit cell) and the more recent results by Kropp et al. [64] (DFT+U, $c(2 \times 2)$ unit cell) are consistent with that finding. Moreover, Kropp et al. [64] indicated that subsurface oxygen vacancies are notoriously less stable than surface ones (by about $1.8 \mathrm{eV}$ ). However, Wu et al. [63] have recently showed that a surface vacancy configuration, with one $\mathrm{Ce}^{3+}$ adjacent to the vacancy in the outermost ceria layer, and the other one in a next-nearest neighbor position in a deeper layer, is the most stable structure (DFT $+\mathrm{U}, \mathrm{p}(2 \times 2)$ unit cell), and that the stability of a subsurface vacancy, 
with one next-nearest neighbor $\mathrm{Ce}^{3+}$ in the outermost ceria layer and the other one nearestneighbor in a deeper layer, is comparable to that of the surface vacancy (within $50 \mathrm{meV}$ ).

Given the the inconsistencies and disparity in some results, in this paper we present a systematic study of the stability of near-surface oxygen vacancies and the excess charge localization at the reduced (110) and (100) ceria surfaces for two different vacancy concentrations, and compare with results in the literature, as well as with those for the (111) surface, clarifying the disagreements, and providing important information and insight on the formation of oxygen vacancies at the lowindex ceria facets, which are particularly interesting in catalysis. The results are discussed in terms of energetics and features of the different ceria facets and vacancy structures. Our findings should be useful in the interpretation of the facet-dependent reactivity of ceria nanoparticles with tailored shapes.

\section{METHODS AND MODELS}

Spin-polarized DFT calculations were carried out using the slab-supercell approach [67], with the Vienna Ab-initio Simulation Package (VASP, http://www.vasp.at; version 5.4.4) $[68,69]$. We explicitly treated the Ce $(4 f, 5 s, 5 p, 5 d, 6 s)$ and $O(2 s, 2 p)$ electrons as valence states within the projector augmented wave (PAW) method with a plane-wave cutoff energy of $415 \mathrm{eV}$, whereas the remaining electrons were considered as part of the atomic core. Strong correlation effects due to charge localization were modeled by adding a Hubbard U-like term [70] $\left(U_{\text {eff }}=U\right.$-J, i.e., the difference between the Coulomb $U$ and exchange J parameters, from now on referred to simply as $U$ ) to the Perdew, Burke and Ernzerhof (PBE) generalized gradient approximation (GGA) functional [71]. We used a value of $U=4.5 \mathrm{eV}$ for the $\mathrm{Ce} 4 \mathrm{f}$ states $[44,72]$.

Bulk ceria has a cubic fluorite structure $(F \mathrm{~m} 3 \mathrm{~m})$ with a calculated lattice constant of 5.486 $\AA$. The low-index $\mathrm{CeO}_{2}$ (111), (110) and (100) facets were modeled employing unit cells of two different sizes, namely, $(2 \times 2)$ and $(3 \times 3),(2 \times 2)$ and $(4 \times 2)$, and $p(2 \times 2)$ and $c(2 \times 2)$ periodicities, respectively (see Figures 1 and S1, and Table 1), respectively, with the optimized lattice parameter. The Monkhorst-Pack method [73] has been used to sample the Brillouin zones and the k-meshes used for each structure are included in Table 1. All supercells were set with a vacuum space of at least $12 \AA$.

The bulk-truncated oxygen-terminated (100) surface is polar, and thus surface reconstructions are expected. Different surface terminations with either $\mathrm{Ce}$ or $\mathrm{O}$ atoms in the 
outermost layer have been proposed in studies employing theoretical and experimental approaches [74-76] We considered the relative stability of two of them, namely, an O-terminated (40-4Ce-80 $\cdots 4 \mathrm{Ce}-40$ ), in which fifty percent of the oxygen atoms in the bulk-truncated surface oxygen layer has been removed and placed at the bottom of the slab, and a Ce-terminated ( $2 \mathrm{Ce}-8 \mathrm{O}-4 \mathrm{Ce} \cdots 8 \mathrm{O}-2 \mathrm{Ce}$ ), in which the cerium atoms are those that are equally distributed between the top and the bottom of the slab (hereinafter (100)-O and (100)-Ce, respectively, cf. Figure 1).

All atoms in the bottom atomic layers were fixed, as indicated in Table 1, at their optimized bulk-truncated positions during geometry optimization, whereas the rest of the atoms were allowed to fully relax. Total energies and forces were calculated with a precision of $10^{-6} \mathrm{eV}$ and $10^{-2} \mathrm{eV} / \AA$ for electronic and force convergence, respectively.

Table 1. Characteristics of the different supercells studied.

\begin{tabular}{|c|c|c|c|c|c|c|}
\hline \multirow{2}{*}{$\begin{array}{c}\text { Surface } \\
\text { Unit cell } \\
\text { periodicity }\end{array}$} & \multicolumn{2}{|c|}{ (111) } & \multicolumn{2}{|c|}{ (110) } & \multicolumn{2}{|c|}{$(100)$} \\
\hline & $(2 \times 2)$ & $(3 \times 3)$ & $(2 \times 2)$ & $(4 \times 2)$ & $p(2 \times 2)$ & $c(2 \times 2)$ \\
\hline k-points mesh & $3 \times 3 \times 1$ & $2 \times 2 \times 1$ & $2 \times 3 \times 1$ & $1 \times 2 \times 1$ & $3 \times 3 \times 1$ & $2 \times 2 \times 1$ \\
\hline $\begin{array}{c}\text { No. atomic } \\
\text { layers }\end{array}$ & 12 & 12 & 5 & 5 & 11 & 11 \\
\hline $\begin{array}{c}\text { Relaxed/fixed } \\
\text { layers }\end{array}$ & $9 / 3$ & $9 / 3$ & $4 / 1$ & $4 / 1$ & $8 / 3$ & $8 / 3$ \\
\hline $\mathrm{CeO}_{2}$ units & 16 & 32 & 20 & 40 & 20 & 40 \\
\hline $\begin{array}{l}\mathrm{CeO}_{2} \text { units per } \\
\text { repeat units } \perp \\
\text { to the surface }\end{array}$ & 4 & 9 & 4 & 8 & 4 & 8 \\
\hline $\begin{array}{l}\text { Surface area } \\
\qquad\left(\mathrm{nm}^{2}\right)\end{array}$ & 0.52 & 1.17 & 0.85 & 1.70 & 0.60 & 1.20 \\
\hline $\begin{array}{l}\text { Molecular } \\
\text { Density } \\
\left(\mathrm{CeO}_{2} / \mathrm{nm}^{2}\right)\end{array}$ & & & & & & \\
\hline
\end{tabular}

Surface energies $(\gamma)$ were calculated as:

$$
\begin{gathered}
\gamma=\gamma^{\text {unrelax }}+\gamma^{\text {relax }} \\
\gamma^{\text {unrelax }}=\frac{1}{2 S}\left[E_{\text {slab }}^{\text {unrelax }}-m \cdot E_{\text {bulk }}\right]
\end{gathered}
$$




$$
\gamma^{\text {relax }}=\frac{1}{S}\left[E_{\text {slab }}^{\text {relax }}-E_{\text {slab }}^{\text {unrelax }}\right]
$$

where $S$ is the surface area of the surface unit cell, $m$ is the number of unit cells with $\mathrm{CeO}_{2}$ composition in the slab, $E_{\text {bulk }}$ is the energy of bulk $\mathrm{CeO}_{2}$ per formula unit, and $E_{\text {slab }}^{\text {relax }}$ and $E_{\text {slab }}^{\text {unrelax }}$ are the total energies of the relaxed and unrelaxed supercells, respectively. Thus, a lower surface energy corresponds to a more stable surface.

(a)
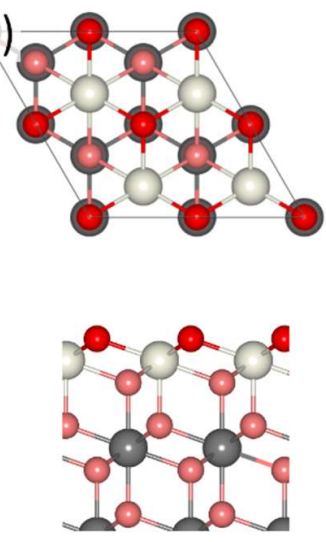
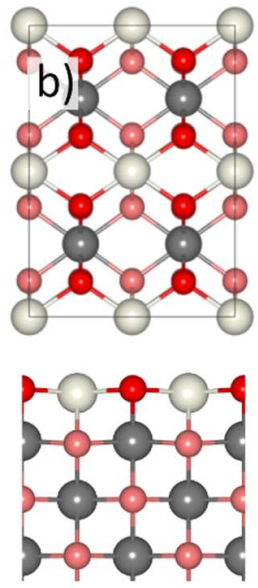
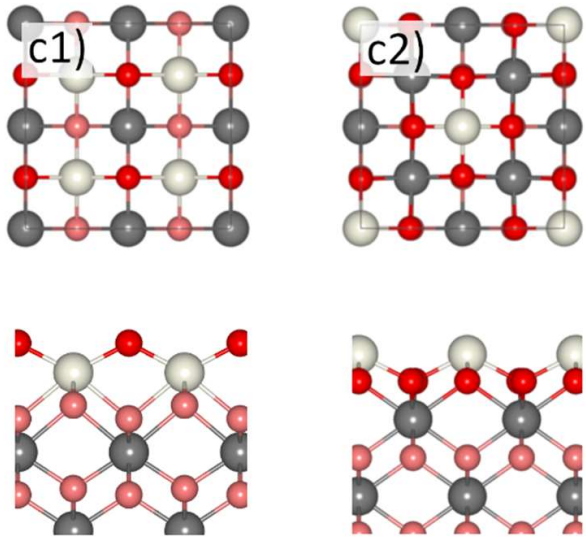

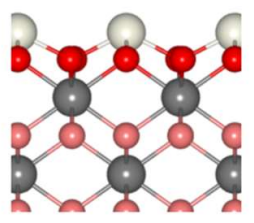

Figure 1. Top and side views of the unrelaxed ceria surfaces: a) $(2 \times 2)-(111), b)(2 \times 2)-(110)$ and, c1) $p(2 \times 2)-(100)$ O-terminated, and c2) p(100) Ce-terminated. Color code: Ce (O) atoms in the outermost layer are white (red) whereas those in deeper layers are gray (light red). This color code is used in all subsequent figures. The images were created employing the VESTA software [77].

Reduced surfaces were modeled by removing one oxygen atom from the surface or subsurface layers. Hereinafter, a capital letter $A(B)$ denotes a surface (subsurface) vacancy, $V^{A / B}$. For all three $p(2 \times 2)$ surfaces, the shortest vacancy-vacancy distance is $776 \mathrm{pm}$, before lattice relaxation. In the larger cells, the corresponding distances are comparable, i.e., 1164, 1097, and 1098 pm for the (111), (110), and (100) facets, respectively. We define the oxygen vacancy concentration as $\Theta_{O_{\text {vac }}}^{\mathrm{A} / \mathrm{B}}=N_{\mathrm{v}} / N^{\mathrm{A} / \mathrm{B}}$, where $N_{\mathrm{v}}$ and $N^{\mathrm{A}}\left(N^{\mathrm{B}}\right)$ are the number of surface (subsurface) vacancies in the reduced layer and the total number of oxygen atoms in a single non-reduced oxygen atomic layer of the same cell, respectively. Therefore, the removal of a surface or subsurface oxygen atom from the $p(2 \times 2)$ surfaces corresponds to $\Theta_{O_{\text {vac }}}^{A}\left(\Theta_{O_{\text {vac }}}^{B}\right)=1 / 4(1 / 4), 1 / 8(1 / 8)$ and $1 / 4(1 / 8)$ for the (111), 
(110) and (100) facets, respectively. In the case of the larger cells, the corresponding concentrations are $\Theta_{O_{\text {vac }}}^{\mathrm{A}}\left(\Theta_{\mathrm{O}_{\mathrm{vac}}}^{\mathrm{B}}\right)=1 / 9(1 / 9), 1 / 16(1 / 16)$ and 1/8 (1/16).

The formation of a neutral oxygen vacancy in ceria is accompanied by the formation of two nearby $\mathrm{Ce}^{3+}$ polarons, which result from the transfer of two electrons originally residing in $2 p$ states of the missing oxygen ion to $\mathrm{Ce} 4 \mathrm{f}$ states of two cations. In order to inspect different configurations of the reduced $\mathrm{Ce}^{3+}$ sites, a two-step relaxation procedure was applied. In the first step, we replaced two selected $\mathrm{Ce}^{4+}$ by $\mathrm{La}^{3+}$ ions (with a larger ionic radii) per $\mathrm{O}$ vacancy and performed non-spinpolarized calculations. The so-obtained relaxed structure was further optimized using the regular $\mathrm{Ce}^{4+}$ PAW potentials. We limit the discussion to high-spin states because the difference between these states and any other spin state is generally less than $0.01 \mathrm{eV}$. The oxidation state of a given $\mathrm{Ce}$ ion $\left(\mathrm{Ce}^{4+}\right.$ or $\left.\mathrm{Ce}^{3+}\right)$ was determined by considering its local magnetic moment (the difference between up and down spins on the ion), which can be estimated by integrating the site- and angular momentum-projected spin-resolved density of states over spheres with radii chosen as the Wigner-Seitz radii of the PAW potentials. The magnetic moment of the $\mathrm{Ce}^{4+}\left(4 f^{0}\right)$ and $\mathrm{Ce}^{3+}\left(4 f^{1}\right)$ ions is 0 and $\sim 1 \mu_{\mathrm{B}}$, respectively, because the occupation of the Ce $f$ states is 0 and $\sim 1$, respectively.

The oxygen vacancy formation energy $\left(E_{f}\right)$ was calculated as

$$
\begin{gathered}
E_{f}=E_{\mathrm{Ce}_{\mathrm{n}} \mathrm{O}_{(2 \mathrm{n}-1)}}+\frac{1}{2} E_{\mathrm{O}_{2}}-E_{\mathrm{CeO}_{2}} \\
E_{f}=E_{f}^{\text {unrelax }}-\Delta E_{f}^{\text {relax }}
\end{gathered}
$$

where $E_{\mathrm{Ce}_{\mathrm{n}} \mathrm{O}_{(2 n-1)}}$ is the energy of the cell with a vacancy, $E_{\mathrm{O}_{2}}$ is the energy of the triplet $\mathrm{O}_{2}$ molecule in gas phase, and $E_{\mathrm{CeO}_{2}}$ is the energy of the unreduced cell. $E_{f}$ can also be written as the difference of two terms $E_{f}^{\text {unrelax }}$ and $\Delta E_{f}^{\text {relax }}$, where $E_{f}^{\text {unrelax }}$ is the energy required to remove an oxygen atom with fix structure, and $\Delta E_{f}^{\text {relax }}(>0)$ is the energy gained from structural relaxations in the presence of the oxygen vacancy. 
(111)
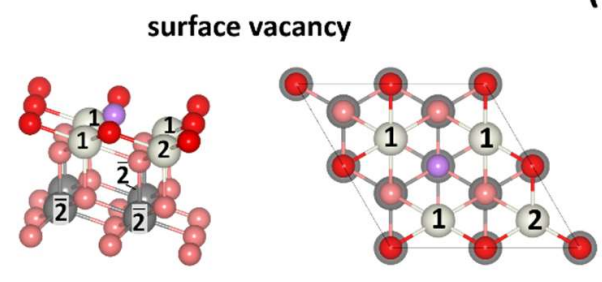

(110)

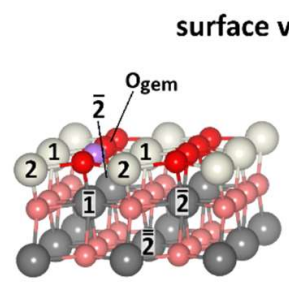

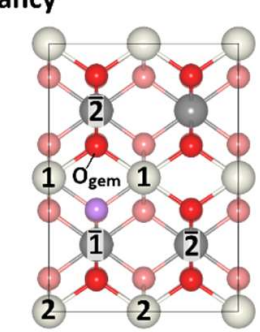

(100) subsurface vacancy
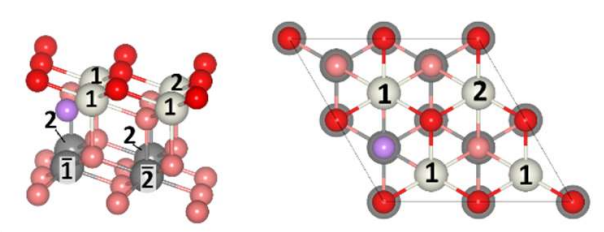

surface vacancy
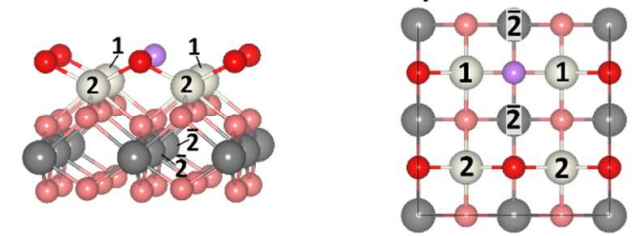

subsurface vacancy 02
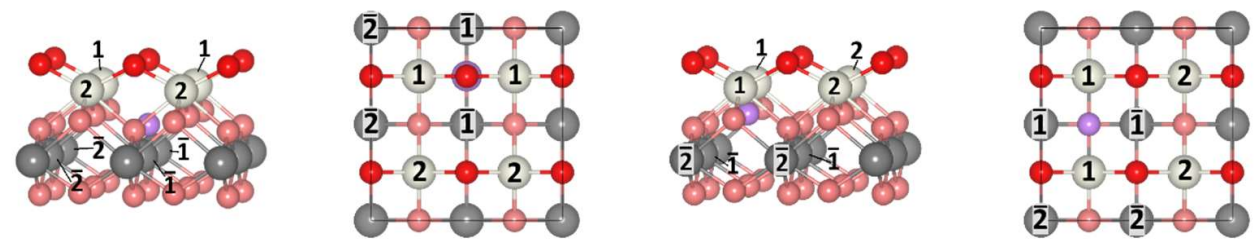

Figure 2. Top and side views of the unrelaxed and reduced (111), (110) and (100) O-terminated lowindex ceria surfaces, with $(2 \times 2),(2 \times 2)$ and $p(2 \times 2)$ periodicity, respectively. The bottom six, two and six atomic layers of the respective slabs are not shown. Oxygen vacancies are shown in purple. The labeled $\mathrm{Ce}$ atoms denote the order of the neighboring cationic coordination shell with respect to the oxygen vacancy to which they belong ( $\bar{n}$ and $\overline{\bar{n}}$ are in the second and third atomic layer, respectively). $O_{\text {gem }}$ indicate a surface oxygen atom at the (110) surface with a change in coordination upon creation of a surface vacancy (see text). 01 and 02 represent the two inequivalent subsurface oxygen positions at the (100) surface.

\section{RESULTS AND DISCUSSION}

\subsection{Surface stability}


In order to understand the stability of near-surface oxygen vacancies at the different ceria facets, it is important to first consider some structural aspects of the corresponding oxidized surfaces, such as the coordination of surface and subsurface oxygen atoms, $\mathrm{O}-\mathrm{Ce}$ distances, and the relative stability between the facets.

In the (111) and (110) facets, surface oxygen atoms are threefold coordinated and in the (100)-0, they are twofold, whereas the subsurface oxygen atoms in all facets are fourfoldcoordinated (see Figure 1 and Table S1). Calculated surface energies, $\gamma$, with and without lattice relaxations follow the same trend, i.e. $(111)<(110)<(100)-0<(100)-C e$ (Table 2). These results are in good agreement with previous studies. The (100)-0 terminated (relaxed) surface is $0.32 \mathrm{~J} / \mathrm{m}^{2}$ more stable than the (100)-Ce terminated one. Therefore, from now on, we will only focus on the (100)-O terminated surface. It should be mentioned that a mixture of $\mathrm{O}$ - and Ce- terminations can reduce the surface energy. For instance, the calculated surface energy for a 87.5\% [(100)-0]-12.5\% $[(100)-C e]$ mixed termination $\left(c(2 \times 2)\right.$ unit cell) is by $0.03 \mathrm{~J} / \mathrm{m}^{2}$ smaller than that of the pure (100)-0 termination, in agreement with what was observed by Pan et al. [74] with a $75 \%-25 \%$ mixed termination. We will not consider these mixed terminations of the (100) facets in more detail, because it is beyond the scope of this work, and only discuss the (100)-O, hereinafter referred as (100).

Table 2. Surface energy of different $\mathrm{CeO}_{2}$ facets.

\begin{tabular}{|c|c|c|c|c|c|}
\hline \multirow{2}{*}{ Method $^{\mathrm{a}}$} & \multicolumn{4}{|c|}{$\gamma(\mathrm{hkl})\left(\mathrm{J} / \mathrm{m}^{2}\right)$} & \multirow{2}{*}{ Reference } \\
\hline & (111) & (110) & $(100)-0$ & $(100)-\mathrm{Ce}$ & \\
\hline $\mathrm{PBE}+\mathrm{U}(4.5)$ & $0.71(0.73)^{b}$ & $1.00(1.36)^{b}$ & $1.45(2.15)^{b}$ & $1.77(3.15)^{b}$ & This work \\
\hline $\mathrm{PBE}+\mathrm{U}(4.5)$ & 0.72 & 1.06 & - & - & [44] \\
\hline $\mathrm{PBE}+\mathrm{U}(4.5)$ & - & - & $1.68^{\mathrm{c}}$ & $1.78^{\mathrm{c}}$ & [74] \\
\hline $\mathrm{PBE}+\mathrm{U}(4.5)$ & - & - & 1.71 & 1.92 & [75] \\
\hline $\mathrm{PBE}+\mathrm{U}(5)$ & 0.73 & 1.09 & 1.48 & 1.83 & [76] \\
\hline PW91+U(5.0) & 0.68 & 1.01 & 1.41 & - & [46] \\
\hline $\mathrm{PBE}+\mathrm{U}(4.5)$ & 0.68 & - & 1.44 & - & [64] \\
\hline PW91+U(6.3) & 0.60 & 0.96 & - & - & [78] \\
\hline $\mathrm{PBE}+\mathrm{U}(5.0)$ & - & - & 1.48 & 1.89 & [79] \\
\hline PW91 & 0.60 & 1.01 & 1.43 & - & [47] \\
\hline PW91+U(5) & 0.64 & 1.39 & 1.65 & & [80] \\
\hline
\end{tabular}


Table 2 also shows that relaxation contribution to the surface energies, $\Delta \gamma$, increases in the following order: $(111)<(110)<(100)-0$, namely, $0.02 \mathrm{~J} / \mathrm{m}^{2}<0.36 \mathrm{~J} / \mathrm{m}^{2}<0.70 \mathrm{~J} / \mathrm{m}^{2}$, respectively. This trend had previously been reported $[42,47,48,64,76,80]$, with $\Delta \gamma$ values in the 0.01-0.04, $0.18-0.24$, and $0.55-0.74 \mathrm{~J} / \mathrm{m}^{2}$ ranges for the (111), (110) and (100)-0 surfaces, respectively. As Nolan et. al. ${ }^{46}$ already noted, the (111) surface is the one that becomes less stabilized through lattice relaxation, because it is the most compact low-index surface, whereas the (100) surface is the one that gains more stabilization energy, as a consequence of significant rearrangements after cleavage. In geometrical terms, the lattice relaxations produce vertical displacements of the outermost $\mathrm{O}$ and Ce atoms. The calculated displacements (in pm), which we describe as $(\Delta \mathrm{O}, \Delta \mathrm{Ce})_{\mathrm{z}}$ are: $(-6.7,-5.0)_{111}$, $(-10.9,-25.5)_{110}$ and $(-28.8,+6.2,)_{100-0}$, where negative (positive) values indicate an inward (outward) relaxation perpendicular to the surface. As expected, the displacements of the $\mathrm{O}$ and $\mathrm{Ce}$ atoms in deeper layers are smaller, but even so, in the second O layer of the (100) termination a strong rumpling is observed, with half of the subsurface atoms of $O$ moving vertically upwards by +29.1 pm and the other half inwards by $-18.5 \mathrm{pm}$, which can be compared with the smaller displacement of the subsurface $O$ atoms in the (110) surface of $-8.3 \mathrm{pm}$. Moreover, an in-plane displacement of the surface $O$ atoms at the (110) surface of about $7 \mathrm{pm}$ is seen. These displacements correlate with changes in the $\mathrm{O}-\mathrm{Ce}$ bond lengths at the surfaces when compared to the $237.5 \mathrm{pm}$ in the $\mathrm{CeO}_{2}$ bulk (Table S1). The average O-Ce bond length for the surface oxygen atoms are 237, 233 and $219 \mathrm{pm}$ for the (111), (110) and (100)-O facets, respectively. The O-Ce bond length at the (111) facet is similar to that in the bulk, because the magnitude of the inward relaxation of the outermost $\mathrm{O}$ and $\mathrm{Ce}$ atoms is comparable. The 1.7\% compression of the average $\mathrm{O}-\mathrm{Ce}$ bond length in the (110) surface, results from the combination of the different vertical displacements of the surface $\mathrm{O}$ and $\mathrm{Ce}$ atoms with the in-plane displacement of the $\mathrm{O}$ atoms. In the case of the (100) facet, the $7.6 \%$ compression of the average $\mathrm{O}-$ Ce bond length results from the inward and outward vertical displacements of the $\mathrm{O}$ and $\mathrm{Ce}$ atoms, respectively.

\subsection{Oxygen vacancy formation}

For the study of the formation of oxygen vacancies we follow the same notation used by Otero et al. [49], i.e., we will refer to $\mathrm{V}_{n, m}^{\mathrm{A}}$ and $\mathrm{V}_{n, m}^{\mathrm{B}}$ for surface and subsurface oxygen vacancies, respectively, as mentioned above. The pair of subscripts $(n, m)(m \geq n)$ are used to indicate the neighboring cationic coordination shells, with respect to the oxygen vacancy, in which the two 
excess electrons $\left(\mathrm{Ce}^{3+}\right)$ are located $(\bar{n}$ and $\bar{m}$, and $\overline{\bar{n}}$ and $\overline{\bar{m}}$, are in the second and third layer, respectively, see Figures 2 and $\mathrm{S} 1 ;\left(n, m^{\prime}\right)$ indicates a non-equivalent configuration to $\left.(n, m)\right)$.

Lattice relaxations are known to be crucial to both the established higher stability of subsurface vacancies at the (111) ceria surface and the localization of the two excess electrons in Ce sites that are not adjacent to the vacancy [37, 43, 52] Therefore, we analyze next their contributions in the cases of the most stable near-surface vacancy structures at the (110) and (100) surfaces, and compare them with those for the (111) surface, using the $p(2 \times 2)$ unit cells.

Table 3. Formation energy of surface $\left(V^{A}\right)$ and subsurface $\left(V^{B}\right)$ oxygen vacancies (with respect to $1 / 2$ $\mathrm{O}_{2}$, in $\mathrm{eV}$ ) at ceria surfaces. $E_{f}^{\text {unrelax }}$ and $E_{f}$ correspond to the values for the unrelaxed and relaxed structures, respectively. $\Delta E_{f}^{\text {relax }}$ corresponds to the energy gained from lattice relaxations upon vacancy formation. $\mathrm{Ce}^{3+}(n, m)$ indicates the positions of the $\mathrm{Ce}^{3+}$ ions in the relaxed structures.

\begin{tabular}{|c|c|c|c|c|c|c|}
\hline \multirow{2}{*}{$\begin{array}{l}\text { Facet } \\
\text { Unit Cell }\end{array}$} & \multicolumn{2}{|c|}{ (111) } & \multicolumn{2}{|c|}{ (110) } & \multicolumn{2}{|c|}{ (100) } \\
\hline & $2 \times 2$ & $3 \times 3$ & $2 \times 2$ & $4 \times 2$ & $p(2 \times 2)$ & $c(2 \times 2)$ \\
\hline Vacancy Type & \multicolumn{6}{|c|}{$\mathrm{V}^{\mathrm{A}}$} \\
\hline$E_{f}^{u n r e l a x}$ & 3.79 & & 3.41 & & 2.61 & \\
\hline$E_{f}$ & 2.22 & 1.96 & 1.29 & 1.06 & 1.78 & 1.35 \\
\hline$\Delta E_{f}^{r e l a x}$ & 1.57 & & 2.12 & & 0.83 & \\
\hline $\mathrm{Ce}^{3+}(n, m)$ & $(2, \overline{2})$ & $(2,2)$ & $(1,2)$ & $(1,2)$ & $(1, \overline{2})$ & $(1,1)$ \\
\hline Vacancy Type & \multicolumn{6}{|c|}{$\mathrm{V}^{\mathbf{B}}$} \\
\hline$E_{f}^{\text {unrelax }}$ & 4.01 & & 4.47 & & 3.86 & \\
\hline$E_{f}$ & 1.81 & 1.76 & 2.17 & 2.29 & 1.75 & 1.82 \\
\hline$\Delta E_{f}^{r e l a x}$ & 2.20 & & 2.30 & & 2.11 & \\
\hline $\mathrm{Ce}^{3+}(n, m)$ & $(2, \overline{2})$ & $(2,2)$ & $(2,2)$ & $(1,2)$ & $(2, \overline{2})$ & $(1,2)$ \\
\hline
\end{tabular}

\subsubsection{Stability trend: unrelaxed structures}

Table 3 shows the calculated vacancy formation energy values obtained from unrelaxed oxygen vacancies, $E_{f}^{\text {unrelax }}$, according to equation (4). The first observation is that for the three 
ceria facets, the formation energy of a surface vacancy is smaller than that of a subsurface vacancy: $E_{f}^{\text {unrelax }}\left(\mathrm{V}^{\mathrm{A}}\right)<E_{f}^{\text {unrelax }}\left(\mathrm{V}^{\mathrm{B}}\right)$. This result is expected $[43,49]$ because fewer $\mathrm{Ce}-\mathrm{O}$ bonds are cut to create surface vacancies ( 2 or 3, cf. Table S1) than subsurface ones (4).

Regarding the dependence of the vacancy formation energies on the ceria facet, two different trends can be observed, depending on the location of the vacancy. For $V^{A}$, the following trend in $E_{f}^{\text {unrelax }}$ is observed: $(100)<(110)<(111)$, whereas for $V^{B}$ is: $(100)<(111)<(110)$. Nolan et al. ${ }^{42,} 48$ already observed that the ordering of stability of unrelaxed surface oxygen vacancies follows that expected from the surface energies [42, 48], i.e., $\gamma(111)<\gamma(110)<\gamma(100)$ (cf. Tables 2 and 3), according to which, less stable surfaces will be more favorable for the formation of oxygen vacancies and thus more catalytically active. This is certainly true for $\mathrm{V}^{\mathrm{A}}$, but not for $\mathrm{V}^{\mathrm{B}}$.

We here further inspect the unrelaxed structures and consider the distribution of the two excess electrons. As mentioned above, lattice relaxations are crucial to the formation of $2 \times \mathrm{Ce}^{3+}$, which leads to the structure stabilization $[43,50]$. However, in the unrelaxed structures, the excess charge is "delocalized" over all nearest-neighbor cations to the vacancies, which are partially reduced, $\mathrm{Ce}^{(4-\delta)+}$. When a surface (subsurface) oxygen vacancy is created without allowing for lattice relaxations, the excess charge is then distributed among 2 (4), 3 (4) and 3 (4) adjacent Ce atoms in the (100), (110) and (111) surfaces, respectively (see Figure S2). Moreover, we recall that reduced Ce ions are larger than the $\mathrm{Ce}^{4+}$, e.g., the average bond length of the eightfold coordinated $\mathrm{Ce}^{4+}$ ions in $\mathrm{CeO}_{2}$ is $2.37 \AA$, whereas that of the sevenfold coordinated $\mathrm{Ce}^{3+}$ ions in $\mathrm{Ce}_{2} \mathrm{O}_{3}$ is $2.50 \AA[43,50]$ This implies that in the unrelaxed structures, the larger $\mathrm{Ce}^{(4-\delta)+}-\mathrm{O}$ bonds are compressed, which increases lattice strain, destabilizing the structures. We here find a correlation between the number of compressed $\mathrm{Ce}^{(4-8)+}-\mathrm{O}$ bonds (NB) and the vacancy formation energy $\left(E_{f}^{\text {unrelax }}\right)$, i.e., the larger $N B$ is, the larger is $E_{f}^{\text {unrel }}$.

The coordination of the outermost Ce atoms is 6 in the pristine (100) and (110) surfaces, and 7 in the (111), whereas it is 8 for Ce atoms in deeper layers for all three facets. In the case of the $\mathrm{V}^{\mathrm{A}}$ surface vacancies at the (100) facet, there are two adjacent $\mathrm{Ce}^{(4-\delta)+}$ ions in the outermost cationic layer and thus there is a total of $2 \times 5=10$ compressed bonds (cf. Figure S2). However, in the (110) facet, out of the three neighboring $\mathrm{Ce}^{(4-\delta)+}$ ions, two are in the first ceria layer and the other one is in the second layer, and therefore NB equals $2 \times 5+7=17$. Finally, in the (111) facet, the three $\mathrm{Ce}^{(4-\delta)+}$ ions are in outermost cationic plane, so that NB is equal to $3 \times 6=21$. This analysis 
indicates that for $V^{A}$ vacancies, NB and $E_{f}^{\text {unrelax }}$ follow the same trend, namely, (100) (NB=10)< $(110)(N B=17)<(111)(N B=18)$.

Inspection of the unrelaxed structures with a $\mathrm{V}^{\mathrm{B}}$ subsurface vacancy (cf. Figure S2), reveals that NB follows the $(100)(N B=24)<(111)(N B=25)<(110)(N B=26)$ trend, which is consistent with the corresponding trend followed by $E_{f}^{\text {unrelax }}$ (cf. Table 3). It is important to note that these results are independent of the unit cell size, since the distribution of the excess charge among adjacent cations to a vacancy in the unrelaxed structures is the same for any cell size (cf. Figures S2 and S3).

\subsubsection{Stability trend: relaxed structures}

Table 3 also shows the lowest vacancy formation energies values for near-surface oxygen vacancies at the three ceria facets, $E_{f}$, which include relaxation effects. The vacancy structures have the excess electrons localized in their energetically preferred cationic sites, which are either in the first or second coordination sphere of the vacant oxygen site. In general terms, the results show that the near-surface oxygen vacancy formation energy $\left(p(2 \times 2)\right.$ cells) follows the trend: $(110)\left(1.29, V^{A}\right)$ $<(100):\left(1.75 / 1.78, V^{B} / V^{A}\right)<(111)\left(1.81 \mathrm{eV}, V^{B}\right)$, which is in accordance with previously reported results $[42,46,48,63,65,81]$ and is not the one expected from the surface energies [cf. $\gamma(111)<$ $\gamma(110)<\gamma(100)$, Table 2]. The near-surface oxygen vacancy stability trend can be related to differences in the packing density of the facets according to which the more compact the facet, the greater is $E_{f}$ (see density of $\mathrm{CeO}_{2}$ units per repeat units $\perp$ to the surface in Table 1). This is in line with the results of Han et al. [51] and Wu et al. [81] that analyzed the near-surface oxygen vacancy stabilization in $\mathrm{CeO}_{2}(111)$ under strain. They both find that tensile strain, involving the decrease in the packing density, is accompanied by the decrease in the energy of vacancy formation.

We further observe that the relaxed surface $V^{A}$ vacancies follow the $(110)<(100)<(111)$ stability trend in relation to the surface facet orientation (cf. $E_{f}$ in Table 3), which is not the same as that discussed above for the corresponding unrelaxed structures (i.e, $(100)<(110)<(111)$, cf. $E_{f}^{\text {unrelax }}$ in Table 3). We note that the stability trend of the $\mathrm{V}^{\mathrm{A}}$ vacancies is in line with previous works $[42,63]$, but actual $E_{f}$ values differ since either the $\mathrm{Ce}^{3+}$ were not located in their preferred positions [42] or the details of the DFT+U methodology used are not identical, or both (Table S2). The fact that the $E_{f}$ and $E_{f}^{\text {unrelax }}$ do not follow the same trend indicates that the surface vacancy induced lattice relaxation effects, $\Delta E_{f}^{\text {relax }}$, that are facet-dependent, can be as significant as to change the stability order (Table 3), with differences between facets of up to about $1.3 \mathrm{eV}$. As 
example, Figure 3 shows the average displacements toward the surface vacant site of the differently coordinated first-neighbor oxygen atoms, i.e., 46, 47 and $152 \mathrm{pm}$ at (111), (100) and (110) surfaces, respectively, which reveal that relaxation effects are important.

$\mathbf{V}^{\mathrm{A}}$

a)

(111)

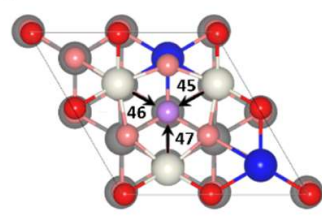

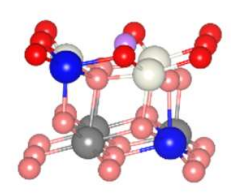

d)

b)

(110)

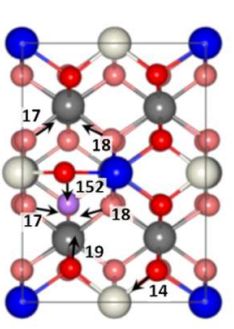

$E_{f}=1.29 \mathrm{eV}$

c)

(100)

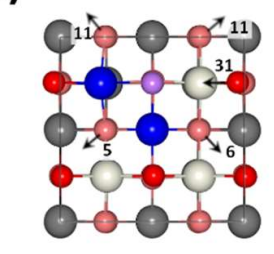

e)
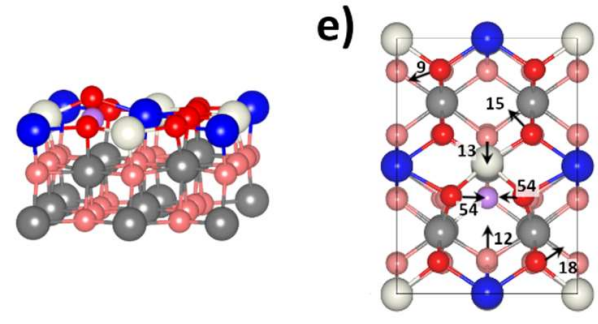

$E_{f}=2.17 \mathrm{eV}$

f)

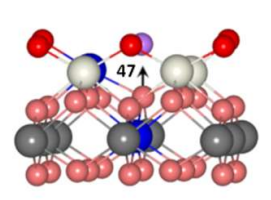

$E_{f}=1.78 \mathrm{eV}$

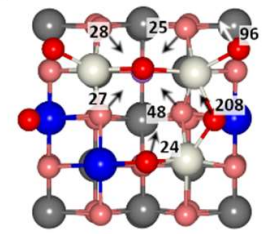

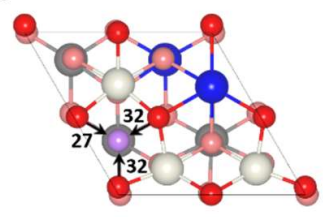

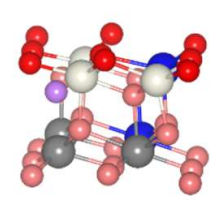

$E_{f}=1.81 \mathrm{eV}$

$\mathbf{V}^{\mathrm{B}}$
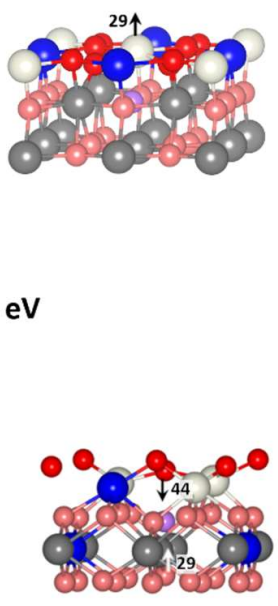

$E_{f}=1.75 \mathrm{eV}$

Figure 3. Top and side views of the most stable surface $\left(V^{A}\right)$ and subsurface $\left(V^{B}\right)$ oxygen vacancy structures for the (111), (110) and (100) ceria facets, with $(2 \times 2),(2 \times 2)$ and $p(2 \times 2)$ periodicity, respectively. The bottom six, two and six atomic layers of the respective slabs are not shown. The oxygen vacancies are shown in purple and the $\mathrm{Ce}^{3+}$ in blue. The vacancy formation energy, $E_{f}$, and selected displacements of the oxygen atoms (in pm), with respect to their positions in the pristine surface, are also indicated.

In the case of the subsurface $\mathrm{V}^{\mathrm{B}}$ vacancies, both $E_{f}$ and $E_{f}^{\text {unrelax }}$ follow the same trend, namely, $(100)<(111)<(110)$. Vacancy induced lattice relaxations effects are also significant and generally even more important than for surface vacancies, but differences between facets are smaller (up to about $0.2 \mathrm{eV}$, Table 3). We note that the stability trend of the $\mathrm{V}^{\mathrm{B}}$ vacancies is not 
consistent with that recently published by Wu et al. [63] [i.e., $(110)<(100)<(111)$ ], which we will address later in the text (section 3.3.2).

Regarding the preferred location of the vacancy, it can be observed that at the least densely packed (110) facet, the $V^{A}$ is more stable than the $V^{B}$, whereas at the most densely packed (111), it is the other way around, and at the (100) facet, both the $V^{A}$ and the $V^{B}$ have similar stability (Table 3 and Figure 3). We observe that these results would be in line with those of Han et al. [51] who reported that the relative stability of surface and subsurface vacancies at the (111) facet depends on strain, with tensile strain favoring the formation of surface vacancies $\left(V^{A}\right)$, whereas subsurface vacancies $\left(V^{B}\right)$ are energetically favored under zero or compressive strain. Furthermore, regarding the location of the $\mathrm{Ce}^{3+}$, we observed that next-nearest neighbor positions to the vacant sites are generally preferred, rather in the outermost layer than in deeper ones (cf. Table 3). However, at the less compact (100) and (110) facets, configurations with nearest neighbor $\mathrm{Ce}^{3+}$ are stable. We will rationalize all of these observations in the following sections.

\subsection{Oxygen vacancies and excess charge localization}

The existence of multiple local minima with respect to the localization of the excess charge may be significant for surface chemistry, since knowing the relevant vacancy structures is important when interpreting results such as the nature of active sites. The preferred cation sites for the localization of the $\mathrm{Ce}^{3+}$ polarons created upon oxygen vacancy formation near the surface of the (111), (110) and (100) ceria facets, and the relative stability of surface and subsurface vacancies, are analyzed below using the $p(2 \times 2)$ surface unit cells.

\subsection{1 $\mathrm{CeO}_{2}(111)$}

As already mentioned, the reduced (111) facet has been the most studied, and the relative stability of near-surface oxygen vacancies and the excess charge localization have been addressed [36-56]. Here, we briefly review the current understanding, as far as relevant for comparison with the reduced (110) and (100) surfaces, and point the reader to works that have compiled most results in the literature $[48,50,55,56]$. The most recent works in the literature (after 2009), independently of the specific implementation of periodic DFT calculations (different unit cell sizes, underlying exchange-correlation functional, $U$ value in $D F T+U$, etc), generally agree that the subsurface vacancy is more stable than the superficial one and that the $\mathrm{Ce}^{3+}$ ions prefer sites that are not adjacent to 
the vacancies, preferably in the outermost cationic layer. The preference of the $\mathrm{Ce}^{3+}$ for the not adjacent sites to the vacancies was explained as being sites in which the $\mathrm{Ce}^{3+}-\mathrm{O}$ bonds are less compressed compared to the adjacent ones (cf. Table S3) [37, 43, 49]. Even though the outermost cerium layer is generally the energetically preferred location of the $\mathrm{Ce}^{3+}$ ions, they would rather be in deeper layers than compressed next to a vacancy, as it is the case with the $(2 \times 2)$ surface unit cell (cf. Table 3) $[43,49,53]$. Therefore, it is noticeable that Wu et al. [57] missed to provide any calculated defect formation energy for $\mathrm{V}_{2, \overline{2}}^{\mathrm{A} / \mathrm{B}}$ configurations using a $(2 \times 2)$ unit cell, but reported a $\mathrm{V}_{1,1}^{\mathrm{A} / \mathrm{B}}$ configurations with both $\mathrm{Ce}^{3+}$ in the first cationic coordination shell.

Lattice relaxations have also been used to explain the preference of vacancies for subsurface sites. Our results for the (111) reduced facet with $(2 \times 2)$ and $(3 \times 3)$ periodicities (Tables 3 and S3, Figures 3 and S4) are in line with the previous knowledge of the system (cf. Table S2). We further here note that the distance between the $\mathrm{Ce}^{3+}$ ions increases by up to $32 \mathrm{pm}$ as the location of the two $\mathrm{Ce}^{3+}$ ions changes when comparing the least with the most stable surface and subsurface vacancy structures $\left(V_{1,1}^{A / B} \rightarrow V_{2,2}^{A / B},(2 \times 2)\right.$ unit cell, Table S3, Figure S4). We observed that a wide spread of values has been reported in the literature for the vacancies formation energies, indicating a strong dependence of the results on the details of the computations (cf. Table S2) $[48,50,55,56$, 82].

\subsection{2 $\mathrm{CeO}_{2}(110)$}

As mentioned in the introduction, for the $\mathrm{CeO}_{2}(110)$ surface, most authors studied only the formation of surface vacancies $[32,33,42,44,58,59,61,63,66]$, and the information on subsurface vacancies is scarce [63]. Moreover, the location of the two $\mathrm{Ce}^{3+}$ ions in the surface vacancy structures considered have varied from being nearest-neighbors in the outermost ceria layer $\left(\mathrm{V}_{1,1}^{\mathrm{A}}\right)$ $[42,44]$ to nearest-neighbors but one in the outermost and one in the second layer $\left(\mathrm{V}_{1, \overline{1}}^{\mathrm{A}}\right)[58,61$, 65], to both $\mathrm{Ce}^{3+}$ in the first ceria layer, one nearest-neighbor and the other one in a next-nearest neighbor position $\left(\mathrm{V}_{1,2}^{\mathrm{A}}\right)[32,33,59,63,66]$. As in the case of the (111) facet, the spread of $E_{f}$ values in the literature is considerable due to differences in the computational details (cf. Table S2), but there is consensus that the latter $\mathrm{Ce}^{3+}$ configuration $\left(\mathrm{V}_{1,2}^{\mathrm{A}}\right)$ is the most stable one and that the result is independent of the size of the unit cell. ${ }^{59}$ However, the reason for the preference of the $\mathrm{V}_{1,2}^{\mathrm{A}}$ 
structure has not yet been explained in sufficient detail. The here calculated stability trend $((2 \times 2)$ unit cell, Table S4) is: $V_{1,1}^{A}(1.88 \mathrm{eV})>V_{\overline{2}, \overline{2}}^{A}, V_{1, \overline{1}}^{A}, V_{2,2}^{A}, V_{2, \overline{2}}^{A}(1.59-1.48 \mathrm{eV})>V_{1,2^{\prime}}^{A}, V_{1,2}^{A}(1.32,1.29 \mathrm{eV})$.

In accordance with the aforementioned importance of lattice relaxations upon vacancy formation for the localization of the excess charge, we here briefly discuss the effect upon creation of surface $V^{A}$ vacancies at the (110) facet. It is the combination of three main reasons what results in the actual stability trend, namely, the general preference of $\mathrm{Ce}^{3+}$ ions to be at the surface, the destabilizing compression of the larger $\mathrm{Ce}^{3+}$ sites in surface sites adjacent to the vacancy, and the repulsive $\mathrm{Ce}^{3+}-\mathrm{Ce}^{3+}$ interaction. The shortest distance between the $\mathrm{Ce}^{3+}$, considering the periodicity of the surface unit cells (Table S4, Figure S5), follows the $V_{1,1}^{A}\left(364\right.$ pm) $<V_{\overline{2}, \overline{2}}^{A}, V_{1, \overline{1}}^{A}, V_{2,2}^{A}, V_{2, \overline{2}}^{A}(380-400$ $\mathrm{pm})<\mathrm{V}_{1,2^{\prime}}^{\mathrm{A}}, \mathrm{V}_{1,2}^{\mathrm{A}}(549-666 \mathrm{pm})$ trend that is generally consistent with that of the stability.

Further inspection of the surface vacancy structures reveals that by moving a $\mathrm{Ce}^{3+}$ from position 1 to $\overline{1}, \mathrm{~V}_{1,1}^{\mathrm{A}} \rightarrow \mathrm{V}_{1, \overline{1}}^{\mathrm{A}}$, not only an increase in the shortest $\mathrm{Ce}^{3+}-\mathrm{Ce}^{3+}$ distance by $36 \mathrm{pm}$ is achieved (Figure S5), but also the initially threefold coordinated surface oxygen ion neighboring the vacancy (the "geminal oxygen ion" [59], $\mathrm{O}_{\text {gem, }}$ Figure 2 ) is pushed outwards to finally occupy a twofold coordinated bridging site between the two top-layer cerium ions next to the vacancy, as previously reported $[58,61]$. Moreover, the average $\mathrm{Ce}^{3+}-\mathrm{O}$ bond length of the atom moving from position 1 to $\overline{1}$ increases by about $11 \mathrm{pm}$, and it is only $7 \mathrm{pm}$ smaller ( $243 \mathrm{pm}, \mathrm{V}_{1, \overline{1}}^{\mathrm{A}}$ in Table S4) than the sevenfold coordinated $\mathrm{Ce}^{3+}$ ions in $\mathrm{Ce}_{2} \mathrm{O}_{3}(250 \mathrm{pm})$. The stronger distortions around the vacant site in the $V_{1, \overline{1}}^{A}$ structure, compared to the $V_{1,1}^{A}$, with the oxygen atoms that are first neighbors to the vacancy moving towards the vacant site and the cerium atoms moving away from the empty negative ion, the better possibility of the $\mathrm{V}_{1, \overline{1}}^{\mathrm{A}}$ structure to relax lattice strain induced by the $\mathrm{Ce}^{3+}$ ions, and the decrease in repulsion between the $\mathrm{Ce}^{3+}$ atoms, result in a $0.33 \mathrm{eV}$ reduction of the vacancy formation energy (from 1.88 to $1.55 \mathrm{eV}$, Table S4). In addition, a further decrease in the repulsive $\mathrm{Ce}^{3+}-\mathrm{Ce}^{3+}$ interaction is achieved by moving a $\mathrm{Ce}^{3+}$ from position $\overline{1}$ to $2, \mathrm{~V}_{1, \overline{1}}^{\mathrm{A}} \rightarrow \mathrm{V}_{1,2}^{\mathrm{A}}$, which is accompanied by and additional energy gain of $0.26 \mathrm{eV}$ (from 1.55 to $1.29 \mathrm{eV}$, Table S4). In the most stable $V_{1,2}^{A}$ configuration, compared it with the $V_{1, \overline{1}}^{A}$ configuration, the germinal oxygen atom has moved $16 \mathrm{pm}$ less with respect to its original position, the two $\mathrm{Ce}^{3+}$ are in the outermost ceria layer and further apart by $266 \mathrm{pm}$, and the average $\mathrm{Ce}^{3+}-\mathrm{O}$ bond length of the atom that moved from position $\overline{1}$ to 2 is comparable (Table S4, Figure S5). The energy gain of $0.59 \mathrm{eV}$ associated to the $\mathrm{V}_{1,1}^{\mathrm{A}} \rightarrow \mathrm{V}_{1,2}^{\mathrm{A}}$ structural changes (Table 4 ) is in very good agreement with that reported by Huang 
et al. [66], where they reported energy differences with respect to the more stable oxygen vacancies.

Table 4. Energy gain by the re-localization of excess charge (in eV) upon creation of surface $\left(\mathrm{V}^{\mathrm{A}}\right)$ and subsurface $\left(V^{B}\right)$ oxygen vacancies at the (111), (110) and (100) ceria facets, with $(2 \times 2),(2 \times 2)$ and $p(2 \times 2)$ periodicity, respectively. The configurations compared are the one obtained without specifying the location of the $\mathrm{Ce}^{3+}$ sites and the most stable one.

\begin{tabular}{|c|c|c|c|}
\cline { 2 - 4 } \multicolumn{1}{c|}{} & $\mathbf{( 1 1 1 )}$ & $\mathbf{( 1 1 0 )}$ & $\mathbf{( 1 0 0 )}$ \\
\hline $\mathbf{V}^{\mathrm{A}}$ & $\mathrm{V}_{1,1}^{\mathrm{A}} \rightarrow \mathrm{V}_{2, \overline{2}}^{\mathrm{A}}$ & $\mathrm{V}_{1,1}^{\mathrm{A}} \rightarrow \mathrm{V}_{1,2}^{\mathrm{A}}$ & $\mathrm{V}_{1,1}^{\mathrm{A}} \rightarrow \mathrm{V}_{1, \overline{2}}^{\mathrm{A}}$ \\
& 0.29 & 0.59 & 0.20 \\
\hline $\mathbf{V}^{\mathrm{B}}$ & $\mathrm{V}_{1,1}^{\mathrm{B}} \rightarrow \mathrm{V}_{2, \overline{2}}^{\mathrm{B}}$ & $\mathrm{V}_{1, \overline{1}}^{\mathrm{B}} \rightarrow \mathrm{V}_{2,2}^{\mathrm{B}}$ & $\mathrm{V}_{\overline{1}, \overline{1}}^{\mathrm{B}} \rightarrow \mathrm{V}_{2, \overline{2}}^{\mathrm{B}}$ \\
& 0.54 & 0.48 & 0.42 \\
\hline
\end{tabular}

To our knowledge, Wu et al. [63] were the only ones that have recently reported on the stability of subsurface vacancies at the $(110)$ facet using a $(2 \times 2)$ unit cell. They found that in the most stable configuration, the $\mathrm{Ce}^{3+}$ are adjacent to the vacancy and below the surface, one in the second and the other in the third ceria layer $\left(1.48 \mathrm{eV}^{63}, \mathrm{~V}_{\overline{1}}^{\mathrm{B}} \overline{\overline{1}}\right.$, Table S2), whereas the second most stable configuration has a next-nearest and a nearest neighbor $\mathrm{Ce}^{3+}$, in the first and third layer, respectively (2.43 $\mathrm{eV}^{63}, \mathrm{~V}_{\overline{1}}, 2$, Table S2). Comparing with their more stable surface vacancy $(1.14 \mathrm{eV}$ [63], $V_{1,2}^{A}$, Table S2), they predicted a greater stability of the latter by $0.34 \mathrm{eV}$. At this point, it is already striking that in their most stable subsurface vacancy configuration both $\mathrm{Ce}^{3+}$ are below the surface.

We have attempted to reproduce the $\mathrm{V}_{\overline{1}}^{\mathrm{B}} \overline{\overline{1}}$ configuration and found that it is unstable, transforming into a surface vacancy with the $\mathrm{V}_{\overline{2}}^{\mathrm{A}} \overline{\overline{2}}$ configuration, after considerable relaxations, particularly of the anions. The fact that for both configurations, $V_{\overline{1}}^{B} \overline{\overline{1}}$ and $V_{\overline{2}}^{A} \overline{\overline{2}}$, they obtained practically the same defect formation energy (1.48 and $1.46 \mathrm{eV}$, respectively [63], cf. Tables S2), could provide an additional indication that the structure $V_{\overline{1}, \overline{1}}^{B}$ does not actually exist. (Note that in the original reference by Wu et al. [63], the $V_{\overline{2}, \overline{\overline{2}}}^{A}$ structure has been mistakenly labelled as $V_{\overline{1}}^{A} \overline{\overline{1}}$ in their Table S2, according to the atomic numbering in their Figure 1a). 
We have considered a number of selected different subsurface vacancy structures with respect to the location of the excess charge $((2 \times 2)$ unit cell, Table S5), and the stability trend for the low-lying vacancy structures is: $V_{\overline{1}, \overline{1}}^{B}(2.97 \mathrm{eV})>V_{1, \overline{1}}^{B}, V_{1, \overline{2}}^{B}, V_{1,2^{\prime}}^{B}, V_{\overline{1}, 2^{B}}^{B}, V_{1,2}^{B}(2.65-2.41 e V)>V_{2,2}^{B}(2.17$ $\mathrm{eV}$ ). As in the case of the surface vacancies, the shortest distance between the $\mathrm{Ce}^{3+}$, considering the periodicity of the surface unit cells (Table S5, Figure S6), follows a trend that is generally consistent with that of the stability, i.e. $V_{1,1}^{B}(377 \mathrm{pm})<V_{1, \overline{1}}^{B}, V_{1, \overline{2}}^{B}, V_{1,2^{\prime}}^{B}, V_{\overline{1}, 2^{B}}^{B}, V_{1,2}^{B}(375-545 \mathrm{pm})<V_{2,2}^{B}(670$ pm). Moreover, in the most stable $\mathrm{V}_{2,2}^{\mathrm{B}}$ configuration (Table S5, Figure $\mathrm{S} 6$ ), the two $\mathrm{Ce}^{3+}$ are in the surface layer, and the average $\mathrm{Ce}^{3+}-\mathrm{O}$ bond lengths are only by 4 and $7 \mathrm{pm}$ smaller than the sevenfold coordinated $\mathrm{Ce}^{3+}$ ions in $\mathrm{Ce}_{2} \mathrm{O}_{3}(250 \mathrm{pm})$.

Similar to the case of the surface vacancies, local structural distortions are strong. In the case of the most stable $\mathrm{V}_{2,2}^{\mathrm{B}}$ subsurface vacancy structure, the two adjacent surface oxygen atoms move by 54 pm (Figure 3, Table S5) and the first Ce neighbors move within a range of approximately 10 to $30 \mathrm{pm}$, with that in the surface layer having the largest displacement (Table S5). In our work, the surface vacancies at the (110) facet are considerable more stable than subsurface ones (by 0.88 $\mathrm{eV}$, Table 3), compared to previously published results (by $0.34 \mathrm{eV}$ ) [63], and both most stable surface and subsurface vacancy structures $\left(V_{1,2}^{A}\right.$ and $V_{2,2}^{B}$, respectively) have the $\mathrm{Ce}^{3+}$ located in the topmost layer and at a distance between them of approximately $670 \mathrm{pm}$.

As mentioned above (sec. 3.3.2), the stability trend of the subsurface vacancies with respect to the orientation of the ceria facet (Table 3), i.e. (100) $\left(V_{2, \overline{2}}^{B}: 1.75 \mathrm{eV}\right)<(111)\left(V_{2, \overline{2}}^{B}: 1.81 \mathrm{eV}\right)$ $<(110)\left(V_{2,2}^{B}: 2.17 \mathrm{eV}\right)$, is not consistent with that recently published by Wu et al. [57, 63] , i.e. (110) $\left(V_{\overline{1}}^{B} \overline{\overline{1}}: 1.48 \mathrm{eV}\right)<(100)\left(V_{2, \overline{2}}^{\mathrm{B}}: 1.67 \mathrm{eV}\right)<(111)\left(V_{2, \overline{2}}^{\mathrm{B}}: 1.75 \mathrm{eV}\right)$. The reason for this inconsistency has to do with the instability, that is, the non-existence, of the $V_{\overline{1}}^{B} \overline{\overline{1}}$ configuration that Wu et al. [63] found it to be more stable, but according to our calculations it becomes a $\mathrm{V}_{\overline{2}}^{\mathrm{A}} \overline{\overline{2}}$.

\subsection{3 $\mathrm{CeO}_{2}(100)$}

The here calculated stability trend for the lower lying surface vacancy structures $(p(2 \times 2)$ unit cell, Table S6) is: $V_{1,1}^{A}(1.98 \mathrm{eV})>V_{1,2^{\prime}}^{A} V_{1,2}^{A}(1.92,1.87 \mathrm{eV})>V_{1, \overline{2}}^{A}(1.78 \mathrm{eV})$. The energy gain associated to the $V_{1,1}^{A} \rightarrow V_{1, \overline{2}}^{A}$ structural changes amounts to $0.20 \mathrm{eV}$ (Table 4). The shortest distance between the $\mathrm{Ce}^{3+}$, considering the periodicity of the surface unit cells (Table S6, Figure S7), follows the $V_{1,1}^{A}(353 \mathrm{pm})<V_{1,2^{\prime}}^{A}(389 \mathrm{pm})<V_{1, \overline{2}}^{\mathrm{A}}(397 \mathrm{pm})$ trend that is generally consistent with that of the 
stability. We note the existence of another $\mathrm{V}_{1,2}^{\mathrm{A}}$-type configuration with $E_{f}=1.87 \mathrm{eV}$ and a $\mathrm{Ce}^{3+}-\mathrm{Ce}^{3+}$ distance of $528 \mathrm{pm}$ (Table S6, Figure S7). A more detailed inspection of the four lower lying energy structures reveals that the percentage of $\mathrm{Ce}^{3+}-\mathrm{O}$ bonds that are shorter than $250 \mathrm{pm}$ (average $\mathrm{Ce}^{3+}-\mathrm{O}$ bond length of the sevenfold coordinated $\mathrm{Ce}^{3+}$ ions in $\mathrm{Ce}_{2} \mathrm{O}_{3}$ ) follows the $\mathrm{V}_{1,1}^{\mathrm{A}}(90 \%$ ) $>V_{1,2^{\prime}}^{A}(81.8 \%)>V_{1,2}^{A}(72.7 \%)>V_{1, \overline{2}}^{A}(69.2 \%)$, which is in line with the calculated stability trend. As an example, we consider here the $\mathrm{V}_{1,1}^{\mathrm{A}}$ configuration in which the two $\mathrm{Ce}^{3+}$ are fivefold coordinated, i.e. a total of ten $\mathrm{Ce}^{3+}-\mathrm{O}$ bonds, out of which nine are shorter than $250 \mathrm{pm}$, i.e. nine out of ten (90\%) of the $\mathrm{Ce}^{3+}-\mathrm{O}$ bonds are compressed. The $\mathrm{Ce}^{3+}$ configuration in the most stable $\mathrm{V}_{1, \overline{2}}^{\mathrm{A}}$ surface vacancy structure agrees with that previously reported by Wu et al. [63]. In the most stable $V_{1, \overline{2}}^{A}$ structure, the two adjacent $\mathrm{Ce}$ ions move away from the vacancy by $18\left(\mathrm{Ce}^{4+}\right)$ and $24\left(\mathrm{Ce}^{3+}\right) \mathrm{pm}$ (Table S6), and the $O$ atom right below the vacancy moves nearly perpendicular to the surface by $47 \mathrm{pm}$ towards the vacant site (Figure 3, Table S6)

When analyzing the subsurface vacancy, it should be noted that there are two types of subsurface oxygen atoms, $O^{B}$ and $O^{B^{\prime}}$, where the former has a neighboring oxygen atom right above in surface layer, whereas the latter does not (cf. Figure 2). We have found that removing the $O^{B}$ atom is considerable easier than the $\mathrm{O}^{\mathrm{B}^{\prime}}$ by up to $1.34 \mathrm{eV}$ (cf. 1.75 and $3.09 \mathrm{eV}$, Table $\mathrm{S} 7$ and 8, respectively). Therefore, the removal of $\mathrm{O}^{\mathrm{B}^{\prime}}$-type oxygen atoms will not be considered further. The here calculated stability trend for the lower lying subsurface vacancy structures $(p(2 \times 2)$ unit cell, Table S7) is: $V_{1,1}^{B}(2.17 \mathrm{eV})>V_{\overline{1}, 2}^{B}(2.14 \mathrm{eV})>V_{1}^{\mathrm{B}}, 2(1.77 \mathrm{eV})>V_{2, \overline{2}}^{\mathrm{B}}(1.75 \mathrm{eV})$.

Once more, the stability trend is generally consistent with that of the shortest $\mathrm{Ce}^{3+}-\mathrm{Ce}^{3+}$ distance (Table S7, Figure S8), i.e. $V_{\overline{1}, \overline{1}}^{B}(373 \mathrm{pm})<V_{\overline{1}, \overline{2}}^{B}(388 \mathrm{pm}) \sim V_{\overline{1}, 2}^{B}(381 \mathrm{pm})<V_{2, \overline{2}}^{B}(392 \mathrm{pm})$. Furthermore, the average $\mathrm{Ce}^{3+}-\mathrm{O}$ bond lengths in the most stable $\mathrm{V}_{2, \overline{2}}^{\mathrm{B}}$ subsurface vacancy structure are the largest among all the configurations considered, namely 258 and $250 \mathrm{pm}$ for the $\mathrm{Ce}^{3+}$ in the second cationic neighboring shell at the first and second cationic plane, respectively. The $\mathrm{Ce}^{3+}$ configuration in the $V_{2, \overline{2}}^{B}$ subsurface vacancy structure, agrees with that previously reported by $\mathrm{Wu}$ et al. [63] (Note that in the original reference by $W u$ et al. [63], the $V_{2, \overline{2}}^{B}$ structure has been mistakenly labelled as $V_{1, \overline{2}}^{B}$ in their Table $S 3$, according to the atomic numbering in their Figure $1 b$ ).

In all of these subsurface vacancy structures, the movements of the neighboring oxygen and cerium atoms towards the vacant site and away from it, respectively, are considerable (Figures 3 and S8, Table S8). Most noticeable is the quasi perpendicular inward displacement of the 
neighboring oxygen atom right above the vacant site in surface layer, which in the case of the most stableV ${ }_{2, \overline{2}}^{\mathrm{B}}$ configuration amounts to $44 \mathrm{pm}$ (Figure 3 , Table S8).

Summarizing our results for the most stable surface $V_{1, \overline{2}}^{A}(1.78 \mathrm{eV})$ and subsurface $V_{2, \overline{2}}^{B}(1.75$ eV) vacancies at the $(100)$ facet with $p(2 \times 2)$ periodicity, we conclude that their stability are comparable, in line with previous results [63].

\subsection{Effect of vacancy concentration}

In this section, we briefly analyze the results on the formation of oxygen vacancies in the near-surface of the (111), (110) and (100) ceria facets using the $(3 \times 3),(4 \times 2)$ and $c(2 \times 2)$ surface unit cells (Figure S1), respectively, which corresponds to a decrease in the vacancy concentration considered so far by a factor of two $\left[\Theta_{O_{\text {vac }}}^{A}\left(\Theta_{O_{\text {vac }}}^{B}\right)=1 / 9(1 / 9), 1 / 16(1 / 16)\right.$ and 1/8 (1/16), respectively].

The interaction between vacancies is repulsive. At the lower vacancy concentration of $1 / 9$, the most stable surface and subsurface configurations at the (111) facet, namely $V_{22}^{A}$ and $V_{2,2}^{B}$, respectively (Figure S4), are by 0.26 and $0.05 \mathrm{eV}$ more stable than the corresponding most stable structures, namely $V_{2, \overline{2}}^{A}$ and $V_{2, \overline{2}}^{B}$, respectively, at the higher vacancy concentration of $1 / 4$ (Tables 3 and S3). Inspection of the near-surface vacancy structures at both concentrations reveals that the average $\mathrm{Ce}^{3+}-\mathrm{O}$ bond lengths, and the shortest distance between the $\mathrm{Ce}^{3+}$, are comparable (Table S3, Figure S4), but at the lower concentration, the $\mathrm{Ce}^{3+}$ ions are located in the generally preferred surface layer, in cationic sites not adjacent to the vacancies. However, at the higher vacancy concentration of $1 / 4$, they would rather be in a deeper layer than next to a vacancy. Furthermore, the relative stability of the subsurface vacancies decreases by $0.21 \mathrm{eV}$ with decreasing vacancy concentration from $1 / 4$ to $1 / 9$ (cf. $0.41 \mathrm{eV}$ and $0.20 \mathrm{eV}$, Table 3).

At the (110) facet, we find that the most stable surface vacancy at the lower vacancy concentration of $1 / 16$ (Table S9, Figure S10), namely $V_{1,2}^{A}$, has the same $\mathrm{Ce}^{3+}$ configuration than the corresponding one at the higher concentration, though the energy cost to form the vacancy is less by $0.23 \mathrm{eV}$ (cf. 1.06 and $1.29 \mathrm{eV}$, Table 3). Inspection of both $V_{1,2}^{A}$ vacancy structures reveals that they are very similar, with an increase in the shortest distance between the $\mathrm{Ce}^{3+}$ by $17 \mathrm{pm}$ (cf. 666 and 683 pm, Table S4, Figure S5 and Table S9, Figure S10, respectively). Concerning the subsurface vacancy, we found that an initial $\mathrm{V}_{2,2}^{\mathrm{B}}$ configuration with $(4 \times 2)$ periodicity, equivalent to that 
obtained with the $(2 \times 2)$ cell (Figure 56$)$, is not stable. After structure optimization, a surface oxygen moves into the vacant subsurface site resulting in the most stable $V_{1,2}^{A}$ surface vacancy structure (Table S9, Figure S10). The most stable subsurface vacancy structure at the lower concentration is $\mathrm{V}_{1,2}^{\mathrm{B}}$ with $E_{f}=2.29 \mathrm{eV}$ (Tables 3 and S10, Figure S11), which is by $0.12 \mathrm{eV}$ more stable than the corresponding $\mathrm{V}_{1,2}^{\mathrm{B}}$ structure with the smaller cell (2.41 eV, Table S5, Figure S6).

Finally, we observe that surface vacancies at the (110) surface at the lower concentration of 1/16 are by $1.23 \mathrm{eV}$ more stable than subsurface ones (cf. 1.06 and $2.29 \mathrm{eV}$, Tables S9 and S10, respectively), which implies that the relative stability of the former increases by $0.35 \mathrm{eV}$ with decreasing vacancy concentration from $1 / 4$ to $1 / 8$ (cf. $0.88 \mathrm{eV}$ discussed in sec. 3.3.2 and $1.23 \mathrm{eV}$ noted here).

At the (100) facet, to the equivalent structure to the most stable surface vacancy $V_{1, \overline{2}}^{A}$ configuration in the smaller $\mathrm{p}(2 \times 2)$ cell with $E_{f}=1.78 \mathrm{eV}$ (Table S6, Figure S7), corresponds a vacancy formation energy that is by $0.33 \mathrm{eV}$ smaller (1.45 eV, Table S11, Figure S12). We have found that other configurations of the $\mathrm{V}_{1,2}^{\mathrm{A}}$ type are comparable in stability with $E_{f}$ in the 1.41-1.44 eV range, and that the most stable configuration is of the $\mathrm{V}_{1,1}^{\mathrm{A}}$ type with $E_{f}=1.35 \mathrm{eV}$ (Table S11, Figure S12), in line with the results by Kropp et. al. [64] At the higher vacancy concentration of $1 / 8$, such a $\mathrm{V}_{1,1}^{\mathrm{A}}$ configuration has a vacancy formation energy of $1.98 \mathrm{eV}$ (Table S6, Figure S7). The larger $\mathrm{c}(2 \times 2)$ unit cell allows for the possibility of better accommodating the lattice strain induced by the formation of the $V_{1,1}^{A}$ vacancy, compared to the smaller $p(2 \times 2)$, as it is reflected by, for example, the larger displacements of the one nearest-neighbor oxygen $(\Delta O)$ atom, right below the vacant site, as well as of the two cerium ( $\Delta \mathrm{Ce}$ ) atom in the outermost ceria plane, with respect to their positions at the pristine surface (cf. Tables S6 and S11 as well as Figures S7 and S12).

Regarding the subsurface vacancy at the $(100)$ facet in the larger $c(2 \times 2)$ cell, to the equivalent structure to the most stable surface vacancy $V_{2, \overline{2}}^{B}$ configuration in the smaller $p(2 \times 2)$ cell with $E_{f}=1.75 \mathrm{eV}$ (Table S7, Figure S8), corresponds a vacancy structure with $E_{f}=1.88 \mathrm{eV}$ (Table S12, Figure S13). Moreover, we found that several initial states such as $V_{1} \frac{B}{1}, 2$ and $V \frac{B}{1}, \overline{1}$, which are stable with the smaller $(2 \times 2)$ cell (Table S7, Figure S8), are unstable and transform into surface vacancy structures, namely, $\mathrm{V}_{\overline{2}}^{\mathrm{A}}, 2$ and $\mathrm{V}_{\overline{2}}^{\mathrm{A}} \overline{2}$, respectively (Table S11, Figure S12). The most stable subsurface vacancy structure at the lower concentration is $\mathrm{V}_{1,2}^{\mathrm{B}}$ with $E_{f}=1.82 \mathrm{eV}$ (Tables 3 and S12, Figure $\mathrm{S} 13$ ), which is by $0.49 \mathrm{eV}$ more stable than the corresponding $V_{1,2}^{B}$ structure with the smaller 
cell (2.31 eV, Table S7, Figure S8). In the $\mathrm{V}_{1,2}^{\mathrm{B}}$ structure, the $\mathrm{Ce}^{3+}$ are located in the outermost cationic plane with the $\mathrm{Ce}^{3+}$ in nearest and next-nearest neighbor positions.

Furthermore, we note that at the (100) facet with the lower vacancy concentration, the surface vacancies are by $0.47 \mathrm{eV}$ more stable than the subsurface ones (Table 3$)$. Employing a $p(2 \times 2)$ surface unit cell, a similar stability for both vacancy types is obtained, and thus the preference for the surface becomes more pronounced as the concentration decreases, as modeled using a $c(2 \times 2)$ cell. This finding clarifies the apparent inconsistencies between previous results on the relative stability of the surface and subsurface vacancies at the $(100)$ facet $[42,58,63,64]$ with results indicating pronounced preference for surface sites (by $1.75 \mathrm{eV}$ ) ${ }^{64}$ or the comparable stability of both sites [63] employing a $c(2 \times 2)$ and $p(2 \times 2)$ unit cell, respectively. By having considered both periodicities, we show that the reason for the disagreement is mainly related to the effect of the concentration of the vacancies. We further observe that the preference for surface sites predicted by Kropp et al. [64] is about 3.7 times larger than our prediction (cf. 1.75 and 0.47 eV). Our calculated surface vacancy formation energy using a c(2×2) cell (cf. $1.35 \mathrm{eV}$ in Table 3 ) is in good agreement with their previously published value (cf. $1.38 \mathrm{eV}$ in ref. [64], as listed in Table S2), but the corresponding values for the subsurface vacancy differ by $1.31 \mathrm{eV}$ (cf. 1.82 in Table 3 and $3.13 \mathrm{eV}$ in ref. [64], as listed in Table S2). We suspect that the discrepancy is related to either differences in the nature of the subsurface oxygen that was removed ( $\mathrm{O} 1$ or $\mathrm{O} 2$, cf. Figure 2 ) or to the localization of the $\mathrm{Ce}^{3+}$, or to both, but no information on the subsurface vacancy structure was provided in ref. [64].

We finally observe that at the two vacancy concentrations here considered, the stability trend in the near-surface vacancy formation energies, in relation to the surface facet orientation, is the same (cf. Table 3), i.e. $(110)<(100)<(111)$.

\section{SUMMARY AND CONCLUSIONS}

We have presented a computational study using the DFT+U methodology of the facetdependent stability of near-surface oxygen vacancies at the low-index (111), (110) and (100) $\mathrm{CeO}_{2}$ surfaces for two different vacancy concentrations, addressing the excess charge localization effects, leading to the formation of $\mathrm{Ce}^{3+}$ polarons. 
The structural properties and stability trend of the pristine surfaces, i.e. $(111)<(110)<$ (100), are in good agreement with previous DFT results. The calculations of the reduced surfaces, with smaller and larger cells, predict that the near-surface vacancy formation energies for comparable vacancy spacings, i.e. $(110)<(100)<(111)$, do not follow the trend of the surface stability of the facets, but that of the packing density, according to which the more compact the facet, the greater is $E_{f}$. The relative preference of vacancies for surface or subsurface sites depends on facet and concentration. The lower concentrations favor a greater stability of surface vacancies at the less densely packed (110) and (100) surfaces, but not at the (111), for which vacancies always prefer the subsurface.

Lattice relaxations occurring upon vacancy formation and the localization of the excess charge are as significant so as to modify various facet-dependent stability trends observed for the unrelaxed vacancy structures, such as that of the surface vacancies and the relative stability of surface and subsurface vacancies. For instance, upon lattice relaxations, the creation of surface vacancies is more facile at the (110) facet, whereas it is at the (100) facet for the unrelaxed structures. Moreover, subsurface vacancies become more stable than surface ones at the (111) facet, in contrast to the results of the unrelaxed structures.

Finding the relevant low-lying vacancy structures is challenging, because of the existence of multiple local minima with respect to the sites on which $\mathrm{Ce}^{3+}$ polarons form. We find that the way for these electrons to distribute is facet dependent and is determined by the general preference of $\mathrm{Ce}^{3+}$ ions to be at the surface, the destabilizing compression of the larger $\mathrm{Ce}^{3+}$ sites in sites adjacent to the vacancy, and the repulsive $\mathrm{Ce}^{3+}-\mathrm{Ce}^{3+}$ interaction. The current work reveals that the stability trends of near-surface vacancies generally correlate with those of the shortest $\mathrm{Ce}^{3+}-\mathrm{Ce}^{3+}$ distances, considering the periodicity of the structures, whereby the shorter the $\mathrm{Ce}^{3+}-\mathrm{Ce}^{3+}$ distance, the greater is $E_{f}$. Furthermore, we have observed that the $\mathrm{Ce}^{3+}$ ions are preferably located in the outermost cationic plane in sites not adjacent to the vacancies. This is particularly true for the most densely packed (111) facet, but at the less compact (110) and (100) facets, configurations with nearest neighbor $\mathrm{Ce}^{3+}$ are stable. The gain in energy through the re-localization of the excess charge when the most stable structures are compared with those obtained without having specified the location of the $\mathrm{Ce}^{3+}$ sites, can be as much as $0.6 \mathrm{eV}$.

From the calculations performed in this work for the higher vacancy concentration, we learn that the higher stability of surface vacancies at the (110) facet compared to subsurface ones, is about 2.6 times larger than previously predicted [63], and that the stability trend of subsurface 
vacancies is: $(100)<(111)<(110)$, and therefore the one recently reported $[57,63]$ is not correct. We further show that for the higher vacancy concentration, the electrons resulting from the formation of a subsurface vacancy at the (110) facet $\left(\Theta_{\mathrm{O}_{\mathrm{vac}}}^{\mathrm{B}}=1 / 8\right)$, do not localize on adjacent cations below surface $\left(V^{B}, \overline{1}\right)$, but that relaxations leads to a global minimum structure with the electrons localized on next-nearest neighbor sites in the surface layer instead $\left(V_{2,2}^{B}\right)$. At a lower subsurface vacancy concentration $\left(\Theta_{O_{\text {vac }}}^{\mathrm{B}}=1 / 16\right), \mathrm{Ce}^{3+}$ can occupy an adjacent site to the vacancy $\left(V_{1,2}^{B}\right)$, but never below the surface.

Inconsistencies were also found with respect to the relative stability of the surface and subsurface vacancies at the (100) surface with the lower concentration of the vacancies, ${ }^{64}$ with results indicating the surface preference by $\sim 1.8 \mathrm{eV}$ [64]. We produce computational evidence that at the lower vacancy concentration, the surface sites are preferred by $\sim 0.5 \mathrm{eV}$. We think that the disagreement in the relative stability of the vacancies is related to either differences in the nature of the subsurface oxygen that was removed or to the localization of the $\mathrm{Ce}^{3+}$, or to both. In conclusion, our thorough study reveals how important is the localization of the excess charge for the interpretation of the oxygen vacancy structures of ceria surfaces and helps us to resolve some inconsistencies in the literature. These results may also have significant implications in many applications of shaped-controlled ceria-based nanostructures.

\section{ASSOCIATED CONTENT}

Supporting Information

\section{ACKNOWLEDGEMENTS}

This project received funding from MICINN-Spain (RTI2018-101604-B-I00) and the European Union's Horizon 2020 research and innovation programme under the Marie Skłodowska-Curie grant agreement No 832121. Computer time provided by the BIFI-ZCAM, the RES (Red Española de Supercomputación) resources at MareNostrum 4 (BSC, Barcelona), Altamira (IFCA, Cantabria), and LaPalma (IAC, La Palma) nodes, and the DECI resources at Finis Terrae II based in Spain at CESGA, with the support from PRACE aislb, is acknowledged. We thank Thomas Kropp and Joachim Paier for discussions on their published data.

\section{Data availability statement}


The DFT data that support the findings of this study are available in Materials Cloud \{https://www.materialscloud.org/home\} with the identifier doi: 10.24435/materialscloud:ax-hy. The repository contains the calculations described in tables S3 to S12 of the Supporting Information. The data are also available from the corresponding author on reasonable request.

\section{ORCID iDs}

Patricia Pérez Bailac https://orcid.org/0000-0002-9992-1875

Pablo G. Lustemberg https://orcid.org/0000-0003-4058-4023

M. Verónica Ganduglia-Pirovano http://orcid.org/0000-0003-2408-8898

\section{REFERENCES}

1 Trovarelli A 2002 Catalysis by Ceria and Related Materials (Imperial College Press and Distributed by World Scientific Publishing Co.

2 Figueiredo W T, Della Mea G B, Segala M, Baptista D L, Escudero C, Pérez-Dieste V and Bernardi $\mathrm{F}$ Understanding the Strong Metal-Support Interaction (SMSI) Effect in $\mathrm{Cu}_{x} \mathrm{Ni}_{1-\mathrm{x}} / \mathrm{CeO}_{2}(0<$ $\mathrm{x}<1$ ) Nanoparticles for Enhanced Catalysis 2019 ACS Appl. Nano. Mat. 2 2559-2573

3 Trovarelli A Catalytic Properties of Ceria and $\mathrm{CeO}_{2}$-Containing Materials 1996 Catal. Rev. 38 439-520

$4 \quad$ Vita $\mathrm{A}$, Pino L, Cipitì F, Laganà $\mathrm{M}$ and Recupero V Structured Reactors as Alternative to Pellets Catalyst for Propane Oxidative Steam Reforming 2010 Int. J. Hydrog. Energy 35 9810-9817

5 Lo Faro M, Vita A, Pino L and Aricò A S Performance Evaluation of a Solid Oxide Fuel Cell Coupled to an External Biogas Tri-Reforming Process 2013 Fuel. Process. Techno. 115 238-245

6 Lustemberg P G, Feria L and Ganduglia-Pirovano M V Single Ni Sites Supported on $\mathrm{CeO}_{2}(111)$ Reveal Cooperative Effects in the Water-Gas Shift Reaction 2019 J. Phys. Chem. C 123 7749-7757

$7 \quad$ Liu $\mathrm{Z}$ et al. Dry Reforming of Methane on a Highly-Active Ni-CeO${ }_{2}$ Catalyst: Effects of MetalSupport Interactions on C-H Bond Breaking 2016 Angew. Chem. Int. Ed. Engl. 55 7455-7459

$8 \quad$ Liu Z et al. In Situ Investigation of Methane Dry Reforming on Metal/Ceria(111) Surfaces: Metal-Support Interactions and C-H Bond Activation at Low Temperature 2017 Angew. Chem. Int. Ed. Engl. 56 13041-13046

9 Lustemberg P G, Zhang F, Gutiérrez R A, Ramírez P J, Senanayake S D, Rodriguez J A and Ganduglia-Pirovano $M \vee$ Breaking Simple Scaling Relations through Metal-Oxide Interactions: Understanding Room-Temperature Activation of Methane on $\mathrm{M} / \mathrm{CeO}_{2}$ ( $\mathrm{M}=\mathrm{Pt} \mathrm{Ni}$ or $\mathrm{Co}$ ) Interfaces 2020 J. Phys. Chem. Lett. 11 9131-9137

10 Lustemberg P G et al. Direct Conversion of Methane to Methanol on Ni-Ceria Surfaces: Metal-Support Interactions and Water-Enabled Catalytic Conversion by Site Blocking $2018 \mathrm{~J}$. Am. Chem. Soc. $1407681-7687$

11 Lustemberg P G, Ramírez P J, Liu Z, Gutiérrez R A, Grinter D G, Carrasco J, Senanayake S $D$, Rodriguez J A and Ganduglia-Pirovano M V Room-Temperature Activation of Methane and Dry 
Re-forming with $\mathrm{CO}_{2}$ on Ni-CeO 2 (111) Surfaces: Effect of $\mathrm{Ce}^{3+}$ Sites and Metal-Support Interactions on C-H Bond Cleavage 2016 ACS Catal. 6 8184-8191

12 Martínez-Munuera J C, Zoccoli M, Giménez-Mañogil J and García-García A Lattice Oxygen Activity in Ceria-Praseodymia Mixed Oxides for Soot Oxidation in Catalysed Gasoline Particle Filters 2019 Appl. Catal. B 2019245 706-720

13 Pan C, Zhang D and Shi L CTAB Assisted Hydrothermal Synthesis Controlled Conversion and $\mathrm{CO}$ Oxidation Properties of $\mathrm{CeO}_{2}$ Nanoplates Nanotubes and Nanorods $2008 \mathrm{~J}$. Solid State Chem. 181 1298-1306

14 Jasinski P, Suzuki T and Anderson H U Nanocrystalline Undoped Ceria Oxygen Sensor 2003 Sensors Actuat. B-Chem. 95 73-77

15 Kulik T, Palianytsia B and Larsson M Catalytic Pyrolysis of Aliphatic Carboxylic Acids into Symmetric Ketones over Ceria-Based Catalysts: Kinetics Isotope Effect and Mechanism 2020 Catal. 10179

16 Xu C and Qu X Cerium Oxide Nanoparticle: A Remarkably Versatile Rare Earth Nanomaterial for Biological Applications 2014 NPG Asia Mater. 6 e90

17 Pulido-Reyes G, Rodea-Palomares I, Das S, Sakthivel T S, Leganes F, Rosal R, Seal S and Fernandez-Pinas F Untangling the Biological Effects of Cerium Oxide Nanoparticles: The Role of Surface Valence States 2015 Sci. Rep. 515613

18 Melchionna M and Fornasiero P The Role of Ceria-Based Nanostructured Materials in Energy Applications 2014 Mat. Today 17 349-357

19 Huang W and Gao Y Morphology-Dependent Surface Chemistry and Catalysis of $\mathrm{CeO}_{2}$ Nanocrystals 2014 Catal. Sci. Technol. 4 3772-3784

20 Trovarelli A and Llorca J Ceria Catalysts at Nanoscale: How Do Crystal Shapes Shape Catalysis? 2017 ACS Catal. 7 4716-4735

21 Hu Z, Liu X, Meng D, Guo Y, Guo Y and Lu G Effect of Ceria Crystal Plane on the Physicochemical and Catalytic Properties of Pd/Ceria for CO and Propane Oxidation 2016 ACS Catal. $62265-2279$

22 Vilé G, Colussi S, Krumeich F, Trovarelli A and Pérez-Ramírez J Opposite Face Sensitivity of $\mathrm{CeO} 2$ in Hydrogenation and Oxidation Catalysis 2014 Angew. Chem. Int. Ed. 53 12069-12072

23 Cao Y, Zhao L, Gutmann T, Xu Y, Dong L, Buntkowsky G and Gao F Getting Insights into the Influence of Crystal Plane Effect of Shaped Ceria on Its Catalytic Performances 2018 J. Phys. Chem. C 122 20402-20409

$24 \quad \mathrm{LiC}$ et al. Shape-Controlled $\mathrm{CeO}_{2}$ Nanoparticles: Stability and Activity in the Catalyzed $\mathrm{HCl}$ Oxidation Reaction 2017 ACS Catal. 7 6453-6463

25 Ren C, Yang R, Li Y, and Wang H Modulating of Facets-Dependent Oxygen Vacancies on Ceria and its Catalytic Oxidation Performance 2019 Res. Chem. Intermed. 45 3019-3032

26 Florea I, Feral-Martin C, Majimel J, Ihiawakrim D, Hirlimann C and Ersen O ThreeDimensional Tomographic Analyses of $\mathrm{CeO}_{2}$ Nanoparticles 2013 Cryst. Growth Des. 13 1110-1121

27 Chen J et al. Polar Surface Structure of Oxide Nanocrystals Revealed with Solid-State NMR Spectroscopy 2019 Nat. Commun. 105420

28 Yang C, YuX, Heißler S, Nefedov A, Colussi S, Llorca J, Trovarelli A, Wang Y and Wöll C Surface Faceting and Reconstruction of Ceria Nanoparticles 2017 Angew. Chem. Int. Ed. 56 375-379 29 Crozier P A, Wang R and Sharma R In Situ Environmental TEM Studies of Dynamic Changes in Cerium-Based Oxides Nanoparticles during Redox Processes 2008 Ultramicroscopy 108 14321440

30 Bezkrovnyi O S, Kraszkiewicz P, Ptak M and Kepinski L Thermally Induced Reconstruction of Ceria Nanocubes into Zigzag \{111\}-Nanofacetted Structures and its Influence on Catalytic Activity in CO Oxidation 2018 Catal. Commun. 117 94-98 
32 Ziemba M, Ganduglia-Pirovano M V and Hess C Elucidating the Oxygen Storage-Release Dynamics in Ceria Nanorods by Combined Multi-Wavelength Raman Spectroscopy and DFT $2020 \mathrm{~J}$. Phys. Chem. 11 8554-8559

33 Yang C, Yu X, Heissler S, Weidler P G, Nefedov A, Wang Y, Woll C, Kropp T, Paier J, Sauer $\mathrm{J} \mathrm{O}_{2}$ Activation on Ceria Catalysts-The Importance of Substrate Crystallographic Orientation 2017 Angew. Chem. Int. Ed. Engl. 56 16399-16404

34 Schilling C, Ganduglia-Pirovano M V andHess C Experimental and Theoretical Study on the Nature of Adsorbed Oxygen Species on Shaped Ceria Nanoparticles 2018 J. Phys. Chem. Lett. 9 65936598

35 Ziemba M, Schilling C, Ganduglia-Pirovano M V and Hess C Toward an Atomic-Level Understanding of Ceria-Based Catalysts: When Experiment and Theory Go Hand in Hand 2021 Acc. Chem. Res. 54 2884-2893

36 Esch F, Fabris S, Zhou L, Montini T, Africh C, Fornasiero P, Comelli G, Rosei R Electron Localization Determines Defect Formation on Ceria Substrates 2005 Science 309752

37 Jerratsch J F, Shao X, Nilius N, Freund H J, Popa C, Ganduglia-Pirovano M V, Burow A M and Sauer J Electron Localization in Defective Ceria Films: A Study with Scanning-Tunneling Microscopy and Density-Functional Theory 2011 Phys. Rev. Lett. 106246801

38 Torbrügge S, Reichling $M$, Ishiyama A and Morita S, Custance Ó Evidence of Subsurface Oxygen Vacancy Ordering on Reduced $\mathrm{CeO}_{2}$ (111) 2007 Phys. Rev. Lett. 99056101

39 Kullgren J, Wolf M J, Castleton C W, Mitev P, Briels W J and Hermansson K Oxygen Vacancies Versus Fluorine at $\mathrm{CeO}_{2}(111)$ : A Case of Mistaken Identity? 2014 Phys. Rev Lett 112 156102

40 Olbrich R, Murgida G E, Ferrari V, Barth C, Llois A M, Reichling M and Ganduglia-Pirovano M V Surface Stabilizes Ceria in Unexpected Stoichiometry 2017 J. Phys. Chem. C 121 6844-6851

41 Murgida G E, Ferrari V, Llois A M and Ganduglia-Pirovano M V Reduced $\mathrm{CeO}_{2}(111)$ Ordered Phases as Bulk Terminations: Introducing the Structure of $\mathrm{Ce}_{3} \mathrm{O}_{5} 2018$ Phys. Rev. Mater. 2083609

42 Nolan M, Parker S C and Watson G W The Electronic Structure of Oxygen Vacancy Defects at the Low Index Surfaces of Ceria 2005 Surf. Sci. 595 223-232

43 Ganduglia-Pirovano M V, Da Silva J L and Sauer J Density-Functional Calculations of the Structure of Near-Surface Oxygen Vacancies and Electron Localization on $\mathrm{CeO}_{2}(111) 2009$ Phys. Rev. Lett. 102026101

44 Fabris S, Vicario G, Balducci G, de Gironcoli S and Baroni S Electronic and Atomistic Structures of Clean and Reduced Ceria Surfaces 2005 J. Phys. Chem. B 109 22860-22867

45 Li H-Y, Wang H-F, Gong X-Q, Guo Y-L, Guo Y, Lu G and Hu P Multiple Configurations of the Two Excess $4 f$ Electrons on Defective $\mathrm{CeO}_{2}(111)$ : Origin and Implications 2009 Phys. Rev. B 79 193401

46 Nolan M, Grigoleit S, Sayle D C, Parker S C and Watson G W Density Functional Theory Studies of the Structure and Electronic Structure of Pure and Defective Low Index Surfaces of Ceria 2005 Surf. Sci. 576 217-229

47 Yang Z, Woo T K, Baudin M and Hermansson K Atomic and Electronic Structure of Unreduced and Reduced $\mathrm{CeO}_{2}$ Surfaces: A First-Principles Study 2004 J. Chem. Phys. 120 7741-7749 48 Nolan M 2015 Surface Effects in the Reactivity of Ceria: A First Principles Perspective Catalysis by Materials with Well-Defined Structures ed Wu Z and Overbury S H (Elsevier) 159-192

49 Otero G S, Lustemberg P G, Prado F and Ganduglia-Pirovano M V Relative Stability of NearSurface Oxygen Vacancies at the $\mathrm{CeO}_{2}(111)$ Surface upon Zirconium Doping 2019 J. Phys. Chem. C $124625-638$ 
50 Ganduglia-Pirovano M V 2015 Oxygen Defects at Reducible Oxide Surfaces: The Example of Ceria and Vanadia Defects at Oxide Surfaces ed Jupille J and Thornton G. (Springer) 149-190

51 Han Z-K, Zhang L, Liu M, Ganduglia-Pirovano M V and Gao Y The Structure of Oxygen Vacancies in the Near-Surface of Reduced $\mathrm{CeO}_{2}$ (111) Under Strain 2019 Front. Chem. 7436

52 Murgida $G$ E and Ganduglia-Pirovano $M V$ Evidence for Subsurface Ordering of Oxygen Vacancies on the Reduced $\mathrm{CeO}_{2}(111)$ Surface Using Density-Functional and Statistical Calculations 2013 Phys. Rev. Lett. 110246101

53 Han Z-K, Yang Y-Z, Zhu B, Ganduglia-Pirovano M V and Gao Y Unraveling the Oxygen Vacancy Structures at the Reduced $\mathrm{CeO}_{2}(111)$ Surface 2018 Phys. Rev. Mater. 2035802

54 Zhang D, Han Z-K, Murgida G E, Ganduglia-Pirovano M V and Gao Y Oxygen-Vacancy Dynamics and Entanglement with Polaron Hopping at the Reduced $\mathrm{CeO}_{2}(111)$ Surface 2019 Phys. Rev. Lett. 122096101

55 Ganduglia-Pirovano M V, Hofmann A and Sauer J Oxygen Vacancies in Transition Metal and Rare Earth Oxides: Current State of Understanding and Remaining Challenges 2007 Surf. Sci. Rep. 62 219-270

56 Paier J, Penschke C and Sauer J Oxygen Defects and Surface Chemistry of Ceria: Quantum Chemical Studies Compared to Experiment 2013 Chem. Rev. 113 3949-85

$57 \mathrm{Wu}$, Vegge $\mathrm{T}$ and Hansen $\mathrm{H} \mathrm{A}$ Improved Electrocatalytic Water Splitting Reaction on $\mathrm{CeO}_{2}(111)$ by Strain Engineering: A DFT+U Study 2019 ACS Catal. 9 4853-4861

58 Nolan M Hybrid Density Functional Theory Description of Oxygen Vacancies in the $\mathrm{CeO}_{2}$ (110) and (100) Surfaces 2010 Chem. Phys. Lett. 499 126-130

59 Kullgren J, Hermansson K and Castleton C Many Competing Ceria (110) Oxygen Vacancy Structures: From Small to Large Supercells 2012 J. Chem. Phys. 137044705

60 Yang Z, Yu X, Lu Z, Li S and Hermansson K Oxygen Vacancy Pairs on $\mathrm{CeO}_{2}(110)$ : A DFT+U study 2009 Phys. Lett. A 373 2786-2792

61 Scanlon D O, Galea N M, Morgan B J and Watson G W Reactivity on the (110) Surface of Ceria: A GGA+U Study of Surface Reduction and the Adsorption of $\mathrm{CO}$ and $\mathrm{NO}_{2} 2009 \mathrm{~J}$. Phys. Chem. C 113 11095-11103

62 Yang $Z$, Fu Z, Wei $\mathrm{Y}$ and Hermansson $\mathrm{K}$ The Electronic and Reduction Properties of $\mathrm{Ce}_{0.75} \mathrm{Zr}_{0.25} \mathrm{O}_{2}(110) 2008$ Chem. Phys. Lett. 450 286-291

63 Wu T, López N, Vegge T and Hansen H A Facet-Dependent Electrocatalytic Water Splitting Reaction on $\mathrm{CeO}_{2}:$ A DFT + U Study 2020 J. Catal. 388 1-10

$64 \quad$ Kropp T and Paier J Activity versus Selectivity of the Methanol Oxidation at Ceria Surfaces: A Comparative First-Principles Study 2015 J. Phys. Chem. C 119 23021-23031

65 Song Y-L, Yin L-L, Zhang J, Hu P, Gong X-Q and Lu G A DFT+U Study of CO Oxidation at $\mathrm{CeO}_{2}(110)$ and (111) Surfaces with Oxygen Vacancies 2013 Surf. Sci. 618 140-147

66 Huang Z-Q, Liu L-P, Qi S, Zhang S, Qu Y and Chang C-R Understanding All-Solid FrustratedLewis-Pair Sites on $\mathrm{CeO}_{2}$ from Theoretical Perspectives 2018 ACS Catal. 8 546-554

67 Payne M C, Teter M P, Allan D C, Arias T A and Joannopoulos J D Iterative Minimization Techniques for Ab Initio Total-Energy Calculations: Molecular Dynamics and Conjugate Gradients 1992 Rev. Mod. Phys. 64 1045-1097

68 Kresse $G$ and Furthmüller J Efficient Iterative Schemes for Ab Initio Total-Energy Calculations Using a Plane-Wave Basis Set 1996 Phys. Rev. B 54 11169-11186

69 Kresse $G$ and Joubert D From Ultrasoft Pseudopotentials to the Projector Augmented-Wave Method 1999 Phys. Rev. B 59 1758-1775

70 Dudarev S L, Botton G A, Savrasov S Y, Humphreys C J and Sutton A P Electron-Energy-Loss Spectra and the Structural Stability of Nickel Oxide: An LSDA+U Study 1998 Phys. Rev. B 57 15051509 
71 Perdew J P, Burke K and Ernzerhof M Generalized Gradient Approximation Made Simple 1996 Phys. Rev. Lett. 77 3865-3868

72 Castleton C W, Kullgren J and Hermansson K Tuning LDA+U for Electron Localization and Structure at Oxygen Vacancies in Ceria 2007 J. Chem. Phys. 127244704

73 Monkhorst H J and Pack J D Special Points for Brillouin-Zone Integrations 1976 Phys. Rev. B 13 5188-5192

74 Pan Y, Nilius N, Stiehler C, Freund H-J, Goniakowski J and Noguera C Ceria Nanocrystals Exposing Wide (100) Facets: Structure and Polarity Compensation 2014 Adv. Mater. Interfaces 1 1400404

75 Capdevila-Cortada M and Lopez N Entropic Contributions Enhance Polarity Compensation for $\mathrm{CeO}_{2}$ (100) Surfaces 2017 Nat. Mater. 16 328-334

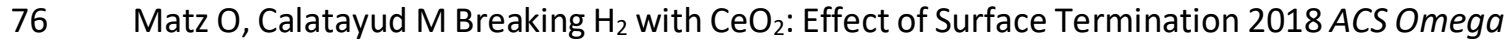
3 16063-16073

77 Momma K and Izumi F VESTA 3 for Three-Dimensional Visualization of Crystal Volumetric and Morphology Data 2011 J. Appl. Crystallography 44 1272-1276

78 Chen H-T, Choi Y M, Liu M and Lin M C A Theoretical Study of Surface Reduction Mechanisms of $\mathrm{CeO}_{2}(111)$ and (110) by $\mathrm{H}_{2} 2007$ Chemphyschem 8 849-855

79 Zhou C Y, Wang D and Gong X Q A DFT+U Revisit of Reconstructed $\mathrm{CeO}_{2}(100)$ Surfaces: Structures Thermostabilities and Reactivities 2019 Phys. Chem. Chem. Phys. 21 19987-19994

80 Marrocchelli D and Yildiz B First-Principles Assessment of $\mathrm{H}_{2} \mathrm{~S}$ and $\mathrm{H}_{2} \mathrm{O}$ Reaction Mechanisms and the Subsequent Hydrogen Absorption on the $\mathrm{CeO}_{2}(111)$ Surface 2012 J. Phys. Chem. C116 24112424

81 Mayernick A D and Janik M J Methane Activation and Oxygen Vacancy Formation over $\mathrm{CeO}_{2}$ and $\mathrm{Zr}$ Pd Substituted $\mathrm{CeO}_{2}$ Surfaces 2008 J. Phys. Chem. C 112 14955-14964

82 Pacchioni G Modeling Doped and Defective Oxides in Catalysis with Density Functional Theory Methods: Room for Improvements 2008 J. Chem. Phys. 128182505 